\title{
Civilisations
}

Revue internationale d'anthropologie et de sciences

humaines

40-1 | 1991

L'Asie et le Pacifique

\section{Les trois dernières années dans le monde et particulièrement en Asie-Pacifique}

Chronologie

Marthe Engelborghs-Bertels

\section{(2) OpenEdition \\ Journals \\ Édition électronique \\ URL : http://journals.openedition.org/civilisations/1689 \\ DOI : 10.4000/civilisations. 1689 \\ ISSN : 2032-0442 \\ Éditeur \\ Institut de sociologie de l'Université Libre de Bruxelles}

Édition imprimée

Date de publication : 1 janvier 1992

Pagination : 293-369

ISBN : 2-87263-063-5

ISSN : 0009-8140

Référence électronique

Marthe Engelborghs-Bertels, « Les trois dernières années dans le monde et particulièrement en AsiePacifique », Civilisations [En ligne], 40-1 | 1991, mis en ligne le 07 juillet 2009, consulté le 23 avril 2019 URL : http://journals.openedition.org/civilisations/1689; DOI : 10.4000/civilisations.1689 
Chronologie dressee par Marthe ENGELBORGHS-BERTELS

\section{LES TROIS DERNIERES ANNEES DANS LE MONDE ET PARTICULIEREMENT EN ASIE-PACIFIQUE}

\section{8}

Abréviations :

ASEAN : Association des nations du Sud Est asiatique

C.C. $=$ Comité central

CEE = Communautée économique européenne

K.M.T. = Kuo Ming Tang, le parti nationaliste au pouvoir à Taiwan

P.C. = parti communiste

R.P. = République populaire

03.01.88 Mort de Tchiang Ching-Kuo à Taiwan

M. Lee Teng hui lui succède à la tête de l'Etat ainsi qu'à la direction du K.M.T., le parti au pouvoir.

16.01.88 Premières élections présidentielles directes en Corée du Sud.

Elles sont gagnées par M. Roh Tae Woo qui entre en fonction le 25.02 .

20-30.01.88 Entretiens près de Paris entre le Présiäent de la coalition tripartite cambodgienne, le Prince Sihanouk et le Premier ministre de Phnom Penh, M. Hun Sen.

03.02.88 La R.P. de Chine et l'Uruguay établissent des relations diplomatiques.

03.02.88 Le K.M.T., au pouvoir à Taiwan, adopte un plan de restructuration qui supprimera l'anomalie des députés élus sur le continent chinois il y a plus de 40 ans.

08.02.88 M. Gorbatchev annonce à Moscou le prochain retrait des troupes soviétiques d'Afghanistan. 
11.02.88 Le Vietnam libère plus de 3.000 détenus de camps de rééducation.

01.03.88 Signature d'un accord entre la CEE et la R.P. de Chine en matière de coopération économique et commerciale 1988-1992

2 et 3/03.88 Le sommet extraordinaire de l'OTAN demande l'ouverture rapide de négociations sur le désarmement conventionnel en Europe afin que cesse la supériorité des forces classiques du Pacte de Varsovic.

03.03.88 Elections parlementaires au Bangladesh remportées par le parti national.

10.03.88 En Indonésie, le Président Suharto est réélu pour un cinquième mandat de 5 ans.

13.03.88 M. D. Lange, Premier ministre de Nouvelle Zélande réaffirme le rejet par son gouvernement de la validité de la théorie de la dissuasion nucléaire.

14.03.88 Echange de feu dans l'archipel des Spratly (Nansha) entre forces chinoises et vietnamiennes.

16.03.88 M. Gorbatchev en visite en Yougoslavie reconnaît l'autonomie des partis et des gouvernements socialistes et la nécessité d'une coopération internationale au-delà des idéologies.

15-19.03.88 Le Secrétaire général du P.C. chinois demande "plus d'ouverture" au comité central de son parti.

21.03.88 Visite aux Etats-Unis du ministre chinois des affaires étrangères.

1.300 étudiants chinois sont rentrés dans leur pays après des études aux Etats-Unis depuis 1987; il en reste 27.000 encore dans des universités américaines.

25.03 au 14.04.88 La première session de la 7ème Assemblée nationale de la R.P. de Chine amende la Constitution de 1982.

Le statut légal des commerçants et entrepreneurs indépendants 
est relevé et le transfert du droit de jouissance de terres est permis.

Un nouveau gouvernement est formé sous la direction de $\mathrm{M}$. Li Peng, Premier ministre.

Un programme de réformes est adopté qui sépare les fonctions du P.C. de celles de la gestion dans les entreprises. M. Yang Shang-kun est élu Président de la République.

M. Deng Xiaoping ne conserve que la présidence des commissions militaires centrales du P.C.C. et de l'Etat.

01.04.88 Un premier accord commercial est conclu entre la R.P. de Chine et la Malaysia.

1-3.04.88 Le Premier ministre de Turquie se rend en Irak pour une mission de médiation entre l'Irak et l'Iran; il discute à Bagdad des développements du Kurdistan iranien où les armes chimiques ont été utilisées.

09.04.88 M. Gorbatchev reçoit à Moscou M. Yasser Arafat; il invite l'OLP à reconnaître l'Etat d'Israel et à prendre en compte les intérêts de sécurité de cet Etat.

04.04.88 Mme C. Aquino, Présidente des Philippines, entame une visite en R.P. de Chine.

12.04.88 Levée de l'état d'urgence au Bangladesh, imposé le 06.12.87.

14.04.88 L'accord sur le retrait militaire soviétique d'Afghanistan est signé à Genève.

A partir du 15.05 .88 , les 115.000 soldats quitteront le pays en neuf mois.

19 au 25.04.88 Visite en R.P. de Chine du Président de la République Grecque, première visite depuis l'établissement des relations diplomatiques intervenues en 1972.

22-28.04.88 Des partisans du mouvement canaque favorable à l'indépendance de la Nouvelle Calédonie (FLNKS) tuent des gendarmes et prennent 27 otages dans l'île d'Ouvéa; 12 seront libérés les 25 et 26 avril. 
05.05. assaut sur la grotte où sont détenus les otages.

12.05. Le dialogue est rétabli avec le gouvernement français.

23.04.88 Signature à Pékin d'un accord sur les échanges culturels et scientifiques entre la R.P. de Chine et la Grande-Bretagne.

24.04.88 La R.P. de Chine proteste contre la déclaration de membres du gouvernement japonais qui nient l'agression japonaise au cours des décennies 1930 et 1940.

24.04.88 Rétablissement de relations entre le P.C. indien et le P.C. chinois après une visite en Chine d'une délégation indienne.

25-27.04.88 Le ministre yougoslave des affaires étrangères effectue une visite en Chine.

26.04.88 Le parti pour la justice et la démocratie du Président Roh Tae Woo obtient 125 des 299 sièges à pourvoir aux élections législatives de Corée du Sud.

Le gouvernement de Sćoul est remanié le 7.5.

26.04.88 L'Arabie Saoudite rompt ses relations diplomatiques avec l'Iran mais annonce que 45.000 pèlerins iraniens pourront se rendre à La Mecque en juillet prochain.

28.04.88 Le projet de loi fondamentale de la région administrative spéciale de Hong Kong qui entrera en vigueur en juillet 1997 est publié simultanément en R.P. de Chine et à Hong Kong.

04.05.88 Le Japon offre une assistance financière à la R.P. de Chine pour son effort de modernisation (191.5 milliards de Yen).

14.05.88 Première réunion du groupe de liaison sino-lusitanien a propos de l'avenir de Macao.

14.05.88 La R.P. de Chine et le Bhutan concluent des conversations a propos de leur frontière commune.

18.05.88 Reddition des extrémistes Sikhs assiégés dans le Temple d'Or d'Amritsar. 
18.05.88 Le "Quotidien du peuple" de Pékin commente l'attitude nouvelle de l'URSS à l'égard de la théorie du socialisme, de la diversité des formes nationales et de l'acceptation de la coexistence de systèmes sociaux differents.

L'URSS abandonne la recherche de la supériorité militaire au profit d'un équilibre stratégique. Elle souhaite réduire les armements militaires et le déploiement militaire à l'étranger comme en témoigne l'évolution en Afghanistan, en Mongolie et dans les pays membres du Pacte de Varsovie.

22.05.88 Le Secrétaire Général du C.C. du parti communiste tchécoslovaque, $M$. Milos Jakes en visite en Chine.

mai 88 Participation de délégués de la R.P. de Chine et de délégués de Taiwan à la réunion annuelle de la Banque asiatique de développement à Manille.

La délégation de Taiwan sera la bienvenue à la prochaine réunion qui aura lieu à Pékin en mai 1989 pour représenter la région "Chine-Taibei", déclare le représentant de la R.P. de Chine.

25.05.88 Le gouvernement victnamien annonce le retrait, avant la fin de 1988, de 50.000 soldats du Cambodge, ce qui représente près de la moitié du contingent qui y est déployé.

29.05.88 Le Premicr ministre du Pakistan est accusé de ne pas maintenir l'ordre et de freiner l'islamisation du pays. Le Président du Pakistan, Zia Ul Haq démet le gouvernement, dissout l'Assemblée nationale et les quatre assemblées locales, promet des élections et le 15.06, décrète que la loi islamique devient la loi suprême.

29.05 au 02.06.88 Le désarmement et les droits de l'homme sont au centre des problèmes discutés au 4ème sommet entre MM. Reagan et Gorbatchev à Moscou.

03.06.88 Visite officielle du Président du Brésil, M. J. Sarney en R.P. de Chine. 
4-12.06.88 Plusieurs centaines d'étudiants sont arrêtés en Coréc du Sud pour avoir voulu rencontrer le 10.06.88 des étudiants de Corée du Nord dans la zone démilitarisée.

05.06.88 Visite officielle en R.P. de Chine du Premier ministre polonais.

07.06.88 Le Parlement du Bangladesh amende la Constitution et déclare l'Islam religion d'Etat.

08.06.88 Une loi de réforme agraire est promulgée aux Philippines; elle oblige les propriétaires fonciers à partager leurs revenus ou leurs propriétés.

13 au $20.06 .8812 \mathrm{ème}$ session des conservateurs sur la normalisation des relations sino-soviétiques à Moscou.

Trois accords de coopération sont signés entre les 2 pays.

15.06.88 Le Gouvernement de la R.P. de Chine rejette la proposition faite par le Dalaï Lama devant le Parlement européen à Strasbourg tendant à faire du Tibet une entité politique autogérée associée à la R.P. de Chine.

22.06.88 M. Do Muoi est nommé Premier ministre du Vietnam.

26.06.88 Les deux communautés concluent un plan de paix en Nouvelle Calédonie. Il sera signé à Paris le 26.08 et prévoit notamment un referendum sur l'indépendance dans dix ans.

30.06.88 Un réexamen de l'action de Staline est en cours en R.P. de Chine; le rapport secret de N. Khrouchtchev au 20ème Congrès du P.C. de l'URSS y est publié.

01.07.88 Le Ministère des Affaires étrangères de la R.P. de Chine déclare que si le Vietnam retire ses troupes du Cambodge, les appuis militaires aux factions du Kampuchea démocratique seront réduits.

03.07.88 Un navire de guerre américain abat un avion civil iranien au dessus du Golfe Persique. 
05.07.88 Le "Guangming Ribao" signale que les dirigeants soviétiques admettent la critique et sa libre expression.

06.07.88 La Ligue Arabe réadmet l'Egypte après 9 années de suspension due à la signature d'un traité de paix séparé avec Israël.

10.07.88 Ouverture près de Jakarta d'une réunion informelle entre représentants des quatre mouvements cambodgiens, du Vietnam, du Laos et des pays d'Asie du Sud Est.

15.07.88 La presse de la R.P. de Chine demande aux dirigeants du K.M.T., au pouvoir à Taiwan, de développer le dialogue par dessus le détroit de Formose.

Pékin souhaite des conversations directes, l'établissement de relations postales et de transport et des échanges commerciaux directs.

15-16.07.88 Le sommet du Pacte de Varsovie propose l'ouverture avant la fin de l'année de négociations sur la réduction des forces armées et des armements classiques en Europe.

18.07.88 L'Iran accepte la résolution du Conseil de Sécurité de l'ONU qui demande le cessez le feu immédiat dans la guerre Iran-Irak.

23.07.88 Le Général Ne Win, à la tête de la Birmanie depuis 1962, démissionne de la présidence du parti unique; son successeur désigné à la direction du parti le 26 et à la direction de l'Etat le 27 est un de ses proches collaborateurs, le Général Sein Lwin.

24-27.07.88 Aux élections générales pour 357 sièges à la Chambre des députés de Thailande, aucun parti ne remporte la majorité. Le 4.8.88, le Général Chatichai Choonhawan devient Premier ministre d'un gouvernement de coalition succédant au Général Prem Tinsulanoda.

25-28.07.88 Réunion informelle à Jakarta, capitale de l'Indonésie, des 3 composantes de la coalition de la République démocratique du Kampuchea et de représentants du gouvernement de Phnom Penh. 
Un groupe de travail est chargé de préparer une autre réunion en 1989.

26.07.88 Les conversations à propos de la révision de l'accord sur les bases militaires américaines aux Philippines sont suspendues.

29.07.88 M. Gorbatchev propose au C.C. du P.C. de l'URSS que les terres en location puissent être attribuées aux paysans pour une durée de 50 ans au maximum.

01.08.88 L'ONU publie des preuves de l'utilisation d'armes chimiques par l'Irak contre des troupes iraniennes et contre des objectifs civils.

03.08.88 La loi martiale est imposée à Rangoon, capitale de la Birmanie. D'importantes manifestations sévèrement réprimées aboutissent à des grèves et à un mouvement massif de la population en faveur de la démocratie.

Après de véritables massacres, le Général Sein Lwin démissionne le 12.8, remplacé par un autre collaborateur de $\mathrm{M}$. Ne Win le $19.8:$ M. Maung Maung.

Le 24.8, la loi martiale est levée à Rangoon et l'armée est renvoyée dans ses casernes.

La ligue pour la démocratie et la paix est formée par $\mathrm{M}$. U Nu, qui a été Premier ministre avant 1962.

08.08.88 Un accord de cessez-le-feu dans le nord de la Namibie est conclu à Genève entre l'Afrique du Sud, l'Angola, Cuba et les Etats-Unis.

Les troupes sud-africaines achèveront leur retrait le 30.08.88.

17.08.88 Le Général Mohamed Zia Ul Haq au pouvoir au Pakistan depuis juillet 1977 meurt dans un accident d'avion. La thèse de l'attentat est retenue par les autorités pakistanaises.

19.08.88 Un premier contact direct est noué entre les deux gouvernements de Corée : 5 députés de chacun des régimes discutent de l'ordre du jour d'une rencontre entre les assemblées nationales du Nord et du Sud. 
20.08.88 Entrée en vigueur du cessez-le-feu entre l'Iran et l'Irak annoncé le 8.8 par M. Javier Perez de Cuellar, Secrétaire général de l'ONU.

26.08.88 Après d'importantes grèves, les autorités polonaises proposent la réunion d'une table ronde à l'opposition.

Les conflits cessent après la rencontre le 31 aout du ministre de l'Intérieur et de M. Walesa.

25-30.08.88 Visite en R.P. de Chine du Premier ministre japonais $M$. Noboru Takeshita.

Le Japon promet 810 milliards de Yen pour le programme de modernisation chinois de 1990-1995.

Un traité sur la protection des investissements en R.P. de Chine est signé le 27.08.88.

En septembre 1988, à une réunion du comité pour l'amitié sino-japonaise du 21ème siècle, le chef de la délégation chinoise considere que le Japon jouera un rôle politique plus important dans un monde qui devient multipolaire mais qu'il doit se garder de toute ressurgence du militarisme.

Les représentants du Japon expliquent la relative faiblesse des investissements nippons en R.P. de Chine par l'instabilité de la politique d'ouverture, l'inefficience des investissements opérés, la confusion des responsabilités et l'étroitesse des contrôles en matière de devise.

28-31.08.88 Conversations sino-soviétiques à Pékin à propos du Cambodge. Le 20.8, le gouvernement de Phnom Penh a été remanié et ne comprend plus de ministère de la coopération économiques avec le Vietnam, le Laos et l'URSS.

03.09.88 Le parti d'action populaire du Premier ministre M. Lee Kuan Yew au pouvoir depuis 1959, remporte 80 des 81 sièges à pourvoir au Parlement de Singapour. M. Lee redevient Premier ministre pour la 8ème fois.

5-8.09.88 Visite du Président de Tchécoslovaquie, M. G. Husak en R.P. de Chine.

Les relations entre les deux Etats et entre les deux P.C. sont complètement normalisées. 
5-10.09.88 Le Secrétaire à la défense des Etats-Unis en R.P. de Chine; son homologue chinois décrit les relations entre les deux pays comme très bénéfiques. Elles montrent la communauté de vues des deux pays à propos du Cambodge.

Les ventes d'armes chinoises à l'étranger sont limitées et insignifiantes; elles continueront à destination du Moyen Orient.

08.09.88 Le Président de la Corée du Nord, M. Kim ll-sung propose une rencontre avec son homologue du Sud pour adopter un pacte de non agression entre les deux pays et explorer les possibilités d'une réunification.

Le 14.9.88, le Président de la Corée du Sud, M. Roh Tae Woo se déclare disposé à une rencontre si l'agenda en est élargi et si la Corée du Nord ne perturbe pas les prochains jeux olympiques.

08.09.88 Visite à Moscou du Président de la République de l'Inde.

10.09.88 Après un mois de protestation et de grèves en Birmanie, le parti au pouvoir promet des élections et le multipartisme.

Le 18.9, les forces armées déposent le Président Maung Maung, abolissent la Constitution et prennent le pouvoir. Un cabinet militaire est constitué le 21.9 sous la direction du Général Saw.

12-16.09.88 M. Gorbatchev en visite dans le territoire de Krasnoïarsk. Il y expose les grandes lignes d'une politique de sécurité en AsiePacifique et en Asie du Sud : amélioration des relations avec la R.P. de Chine, les pays de l'ASEAN et le Japon, participation pleine et entière sur base d'égalité de l'URSS avec les EtatsUnis, baisser le niveau de confrontation, abandonner les facilités de Cam Rank au Vietnam si les Etats-Unis renoncent à leurs bases aux Philippines et réunir une conférence internationale pour créer une zone de paix dans l'Océan Indien.

17.09-02.10 Les 24èmes jeux olympiques se tiennent à Séoul, avec la participation de 160 pays, parmi lesquels figurent l'URSS et la R.P. de Chine. La Corée du Nord, l'Albanie, Cuba et le Nicaragua ont boycotté la réunion. 
19-21.09.88 Visite à Moscou du Président du Conseil d'Etat du Vietnam.

20-21.09.88 La 19ème conférence du Forum du Pacifique Sud se tient dans la capitale de Tonga avec la participation des dirigeants de 15 Etats membres.

24.09.88 Le Premier ministre de Singapour, M. Lee Kuan Yew effectue une visite en R.P. de Chine.

Il rencontre $M$. Deng Xiaoping avec lequel il envisage l'extension de la coopération entre les deux pays.

26.09.88 Le Président des Etats-Unis, M. D. Reagan propose devant l'Assemblée générale de l'ONU la convocation d'une conférence internationale pour l'interdiction totale des armes chimiques.

26 au 30.09.88 3ème réunion plénière du 13ème C.C. du P.C.C. Le rapport présenté par le Secrétaire Général Zhao Ziyang déplace les priorités de la réforme et de la construction vers "l'amélioration de l'environnement économique et la rectification de l'ordre économique".

La lutte contre l'inflation et la réduction de la demande impliquent une stratégie de retranchement a appliquer pendant deux ans au moins, les investissements en fonds fixes seront réduits de $20 \%$ en 1989 et le double système des prix, libres et imposés, est maintenu.

De nouvelles règles sont annoncées pour renforcer la discipline, lutter contre la corruption et les pratiques criminelles, tant au scin du P.C.C. que du personnel de l'Etat. Les groupes dirigeants du P.C.C. seront supprimés dans toutes organisations qui dépendent directement du Conseil d'Etat (le gouvernement central).

29.09.88 Qiao Shih, membre du comité permanent du bureau politique du P.C. chinois rappelle que le centralisme démocratique reste un principe fondamental; les délégations d'autorité ne réduisent en rien la portée des injonctions émises par le pouvoir central. 
01.10.88 M. M. Gorbatchev, secrétaire général du P.C. devient chef d'Etat de l'URSS en remplacement de M. A. Gromyko.

04.10.88 Le Président de la Corée du Sud réitère son offre de rencontre avec M. Kim Il sung. Séoul déclare vouloir lever l'interdiction d'échanges commerciaux avec la Corée du Nord et propose des conversations pour réaliser l'unité économique.

Le 18.10, à l'ONU, M. Roh propose la réunion d'une conférence consultative chargée des moyens d'assurer une paix durable dans le nord-est de l'Asie.

Le 19.10, la Corée du Nord entend que le sommet soit précédé du retrait des troupes américaines de Corée du Sud.

11.10.88 Trois groupes de l'opposition centriste fusionnent en un nouveau parti politique (Janata Dal) en Inde.

11.10.88 Le Président de la République de Finlande effectue une visite officielle en R.P. de Chine. Plusieurs contrats économiques sont signés.

12.10.88 Le vice-premier ministre chinois Yao Yi-lin en visite en R.F.A., invite les hommes d'affaires allemands à augmenter leurs investissements dans son pays.

14.10.88 Le Secrétaire général du P.C. roumain, M. N. Ceaucescu entame une visite en R.P. de Chine.

17.10.88 Les Etats-Unis acceptent de fournir une aide économique et militaire de 481 millions de $\$$ aux Philippines pour continuer à disposer de leurs bases militaires jusqu'en 1991.

25-27.10.88 Les représentants des 16 pays membres du COCOM (comité de coordination des contrôles sur les exportations multilatérales) décident de lever certaines restrictions mises à l'exportation de technologies sensibles (machines-outils, équipement chimique) vers la R.P. de Chine, l'URSS et l'Europe orientale.

31.10-08.11.88 4ème session de la 7ème législature de la R.P. de Chine.

Les autorités centrales doivent renforcer leur contrôle sur les 
hausses des prix et les pressions inflationnistes.

La production de céréales et d'oléagineux doit augmenter; la chute des investissements dans l'agriculture et les déficiences de l'irrigation sont mises en cause.

01.11.88 Accord sino-soviétique sur la définition du tracé de la frontière dans le secteur oriental.

04 au 09.11.88 Visite d'Etat en R.P. de Chine du Président de l'Uruguay. Deng Xiaoping lui déclare que la Chine souhaite faire de ses relations avec l'Amérique latine, un modèle de coopération Sud-Sud.

10 au 24.11.88 Le premier ministre de R.P. de Chine effectue une visite en Thailande, en Australie et en Nouvelle Zélande.

La Chine et la Thailande considèrent que le retrait des troupes vietnamiennes du Cambodge est un préalable à la recherche d'un règlement politique.

Un projet de coopération et d'entreprises conjointes est décidé à Canberra; un accord de protection des investissements est signé entre la R.P. de Chine et la Nouvelle Zélande.

16.11.88. Le parti du peuple de Mme Benazir Bhutto remporte 92 des 207 sièges aux élections législatives du Pakistan. Le 1.12.88, Mme Bhutto est nommée Premier ministre.

17-20.11.88 M. Gorbatchev en visite en Inde assure le Premier ministre M. R. Gandhi que le rapprochement sino-soviétique n'affectera pas les liens de l'URSS avec l'Inde.

21-27.11.88 Le Président de l'Irak, M. S. Hussein annonce que son fils sera jugé pour le meurtre d'un de ses collaborateurs.

Le 27.11, le gouvernement d'Irak autorise de nouveaux partis politiques, accordera l'amnistic pour les crimes politiques et s'engage à respecter les droits de l'homme.

24-25.11.88 Le Premier ministre de Thailande, en visite au Laos administre la preuve de l'amélioration des relations entre les deux pays. 
25.11.88 La R.P. de Chine reconnaît l'Etat indépendant de la Palestine proclamé le 15.11.88 par M. Yasser Arafat. Ce dernier est félicité pour la reconnaissance explicite de l'Etat d'Israël.

29.11-14.12.88 Une délégation sud-coréenne propose à la R.P. de Chine l'établissement de lignes maritimes directes entre les deux pays. Pékin a décidé en septembre 1988 d'ouvrir un bureau commercial non gouvernemental à Sćoul.

1 au 3.12.88 Le ministre chinois des affaires étrangères, M. Qian Qichen discute à Moscou avec son homologue M. E. Chevardnadze du Cambodge et des relations entre l'URSS et la R.P. de Chine.

07.12.88 M. Gorbatchev annonce à l'Assemblée générale de l'ONU une réduction unilatérale de 500.000 hommes des forces militaires de l'URSS. Il abrège son séjour aux Etats-Unis à la suite du violent séisme qui ébranle l'Arménie soviétique le 7/12.

10.12.88 Emeute à Lhassa, la police fait usage de ses armes. Les autorités accusent un petit nombre de séparatistes tibétains.

14/12.88 Les Etats-Unis acceptent d'ouvrir un dialogue avec des représentants de l'OLP.

M. Yasser Arafat précise à Genève qu'il a clairement reconnu le droit d'Israël à vivre en paix et en sécurité et il déclare renoncer totalement au terrorisme.

15.12.88 Visite officielle en R.P. de Chine du Ministre des Affaires Etrangères d'Iran. Les échanges commerciaux sont appelés à se développer entre les deux pays.

19.12.88 Le ministère des affaires étrangères de Pékin accuse les autorités de Taiwan de pratiquer une "diplomatie élastique" en établissant des organisations commerciales, en élevant les établissements non officiels en consulats généraux et en nouant ou renouant des relations diplomatiques avec des pays qui ont de pareilles relations avec la R.P. de Chine. L'objectif est de créer "deux Chine "ou" une Chine et un Taiwan".

La représentation de Taiwan à la Banque asiatique de développement ne constitue pas un précédent. 
19.12.88 M. Ranasinghe Premadasa, Premier ministre de Sri Lanka depuis 1978 remporte l'élection présidentielle organisée dans un extrême tension d'attentats et de répression. Il devient Président de la République le 2.1.1989.

20.12.88 Le C.C. du P.C. de la R.P. de Chine et le Conseil d'Etat consacrent une circulaire à la nécessité de bonnes récoltes de céréales en 1989 et 1990 . Les prix d'achat des céréales par l'Etat sont relevés et le volume des engrais mis en vente à prix stables sera augmenté.

20.12.88 Début de la visite de R.P. de Chine du Premier ministre indien M. Rajiv Gandhi.

L'Inde reste fidèle à sa politique de non ingérence et considère le Tibet comme une région autonome de la R.P. de Chine.

Un groupe de travail conjoint est chargé d'étudier le problème des frontières entre les 2 pays.

22.12.88 Un accord commercial et des notes relatives au commerce frontalier sont signés à Pékin entre la R.P. de Chine et le Laos.

Les deux pays s'accordent mutuellement le traitement de la nation la plus favorisée.

22.12.88 L'Afrique du Sud, l'Angola et Cuba signent au siège des Nations Unies à New York un traité qui prévoit le retrait d'Angola de 50.000 soldats cubains pour le 1.7.1991 et un traité sur l'accession de la Namibie à l'indépendance au début de 1990 après des élections libres à organiser sous l'égide des Nations Unies.

23-29.12.88 Au cours de la session du comité permanent de l'Assemblée nationale de la R.P. de Chine, des députés expriment le besoin de réformes politiques, sociales et culturelles pour une plus grande démocratie.

29.12.88 Le porte parole du Ministère des Affaires étrangères de la R.P. de Chine déclare que les incidents entre étudiants chinois et africains qui ont eu lieu à Nankin n'ont aucune connotation de discrimination raciale. 
30.12.88 Réunion du comité permanent de l'Assemblée populaire nationale de la R.P. de Chine à propos de la réunification de Taiwan. Les relations sont moins tendues, les visites de Taiwanais en R.P. de Chine se multiplient et le commerce indirect se développe. Une zone spéciale sera créce dans laquelle les entrepreneurs taiwanais seront invités à investir.

7-11.01.89 Conférence internationale sur les armes chimiques à Paris. Le Ministre soviétique des affaires étrangères annonce que l'URSS commencera en 1989 la destruction de ses stocks.

Le Ministre des Affaires Etrangères de la R.P. de Chine déclare que son pays ne produit ni ne possedde de pareilles armes. Il s'entretient avec le Ministre israelien des Affaires étrangères auquel il demande d'adopter une attitude réaliste et souple; les représentants permanents à l'ONU de la R.P. de Chine et d'Israël maintiendront le contact.

07.01.89 Mort de l'Empereur Hirohito du Japon.

10.01.89 Le P.C.C. annonce qu'une évaluation de ses membres sera faite chaque année afin de les éduquer et de les contrôler et afin de conserver au P.C.C. le rôle dirigeant.

10.01.89 Le C.C. du P.C. de l'URSS désigne les 100 candidats aux sièges qui lui sont réservés sur les 2.250 du Congrès des députés du peuple et il permet une véritable campagne électorale pour les autres sièges à pourvoir.

14-20.01.89 Le vice ministre vietnamien des Affaires étrangères a des conversations privées à Pékin.

17.01.89 Le C.C. du POUP rétablit le pluralisme syndical en Pologne. 
17-19.01.89 3ème conférence sur la sécurité et la coopération en Europe. Le document final adopté par 34 des 35 pays signataires de l'Acte final de 1975 garantit le respect des droits de l'homme et des libertés fondamentales. Seule, la Roumanie émet des réserves. Les pays membres de l'OTAN et du Pacte de Varsovie entameront en mars 1989 des négociations pour équilibrer les forces conventionnelles en Europe à un niveau réduit.

19.01.89 Le Ministre des Affaires Etrangères de Cuba s'entretient à Pékin avec son homologue chinois et signe un protocole de coopération qui témoigne d'une nouvelle étape dans les relations entre les deux pays après la restauration totale de leurs relations politiques et l'augmentation de leurs échanges.

20.01.89 Le Ministre chinois de la Défense en visite en Thaillande, conclut un accord d'assistance à l'armée thaïlandaise.

20.01.89 M. George Bush prend ses fonctions de 41ème Président des Etats-Unis.

25-27.01.89 Le Premier Ministre du Cambodge, M. Hun Sen est reçu à Bangkok bien que la Thaïlande n'ait pas reconnu son gouvernement.

28.01.89 Mort du Panchen Lama, chef religieux du Tibet et ami dévoué des autorités de la R.P. de Chine.

02.02.89 Le fondateur de la société sud-coréenne Hyundai a conclu en Corée du nord quatre accords de coopération. Le 21.02.89, le gouvernement de Séoul interdit la réalisation de trois de ces projets pour leurs possibles implications militaires.

1-4.02.89 Le Ministre soviétique des Affaires étrangères prépare à Pékin le prochain sommet sino-soviétique. Une déclaration commune exprime un accord pour le retrait total des troupes vietnamiennes du Cambodge en septembre 1989 et des conversations entre les quatre factions cambodgiennes, comme base nécessaire à un juste règlement.

Un contrôle international du retrait des troupes 
vietnamiennes et une réduction des forces militaires, une garantie internationale accordée au statut d'Etat indépendant, neutre et non aligné du Cambodge sont souhaités par l'URSS et la R.P. de Chine.

05.02.89 Le chef d'Etat major du Pakistan annonce l'essai réussi du premier missile sol-sol à longue portée de fabrication pakistanaise.

06.02.89 Ouverture des conversations de la "Table ronde" à Varsovie entre représentants du pouvoir, de l'opposition et de l'Eglise. Le 5 avril 89, un accord prévoit une démocratisation des institutions politiques et l'entrée de l'opposition au Parlement.

10.02.89 Le "Quotidien du peuple" publie un article sur la nécessité de mettre à jour le marxisme.

Les restrictions aux discussions théoriques en R.P. de Chine devraient être abolies; il ne devrait plus exister de zones interdites dans les milieux académiques.

10.02.89 Le principe d'une transition pour le multipartisme est accepté en Hongrie par le C.C. du parti au pouvoir.

11-13.02.89 Visite en R.P. de Chine au Premier ministre pakistanais, Mme B. Bhutto. L'amitié avec la Chine est la pierre angulaire de la politique étrangère du Pakistan.

Le Secrétaire général du P.C.C. Zhao Ziyang demande le développement des relations entre le P.C.C. et le parti du peuple pakistanais dont Mme Bhutto est le président.

14.02.89 L'iman Khomeiny ordonne l'exécution rapide de l'écrivain Salman Rushdie.

14.02.89 Le gouvernement de la R.P. de Chine décide de renforcer le contrôle sur les emprunts contractés à l'étranger afin de ralentir la croissance rapide de la dette extérieure.

15.02.89 Le parti au pouvoir au Sri Lanka remporte la majorité aux élections législatives. Le 03.03.89, M. Wijetunge devient Premier ministre en remplacement de M. Premadasa. 
15.02.89 Les troupes soviétiques ont achevé leur retrait d'Afghanistan, conformément aux accords de Genève du 14.04.88. Un gouvernement afghan est formé en exil au Pakistan et en Afghanistan même, l'état d'urgence est proclamé le 19.02.89.

19-21.02.89 Deuxième réunion informelle sur le Cambodge à Jakarta, sans la participation du président de la résistance, le Prince Sihanouk. La première a eu lieu en juillet 1988.

17-27.02.89 M. E. Chevardnadze effectue une tournée en Syrie, en Jordanie, en Egypte, en Irak et en Iran; au Caire, il s'entretient avec le Ministre israélien des Affaires étrangères et ensuite avec M. Yasser Arafat, le dirigeant de l'O.L.P.

20.02.89 Dans une lettre ouverte au gouvernement de la R.P. de Chine, 33 artistes et intellectuels demandent la libération des prisonniers politiques et la réaffirmation de la liberté de parole garantie par la Constitution.

Le Professeur Fang Lizhi écrit personnellement à M. Deng Xiaoping et demande la création d'un groupe de travail sur l'"amnistie 1989".

Les autorités considèrent ces demandes comme des pressions sur le gouvernement et des ingérences dans le fonctionnement de la justice.

22.02.89 Elections générales aux Iles Salomon : le principal parti au pouvoir depuis 1989 est vaincu. C'est le dirigeant du parti de l'alliance populaire, M. Solomon Mamaloni qui devient Premier ministre.

24.02.89 Les funérailles de l'Empereur du Japon sont l'occasion de rencontres entre dirigeants des 163 pays représentés.

25.02.89 Le Président des Etats-Unis, M. G. Bush effectue une visite de travail en R.P. de Chine.

Les relations entre les deux pays sont jugées satisfaisantes mais les autorités chinoises déplorent les tentatives faites par certains Américains pour influencer la politique chinoise. Les autorités ne permettent pas au Professeur Fang Lizhi d'assister au banquet auquel M. Bush l'avait invité. 
26.02.89 La presse de la R.P. de Chine évoque la masse des travailleurs ruraux qui afflue dans les villes à la recherche d'un emploi.

Le phénomène des migrations internes est agravé par la politique d'austérité et par le système des contrats de responsabilité qui contraint beaucoup de ruraux à s'engager dans des travaux non agricoles.

27.02.89 Le Président G. Bush en Corée du Sud s'engage à maintenir les 43.000 militaires américains dans le pays et plaide pour l'élimination des obstacles aux importations de produits américains en Corée du Sud.

02.03.89 Le Sultan de Perak est élu chef de l'Etat (Roi) de la Malaysie.

03.03.89 Les autorités de Mongolie annoncent leur intention de réduire les forces armées de $50 \%$.

07.03.89 Le Premier ministre de R.P. de Chine, M. Li Peng décrète la loi martiale à Lhassa après des émeutes anti-chinoises au Tibet, à l'approche du 30ème anniversaire de la révolte du 10 mars 1959.

Le Dalai Lama demande à $M$. Deng Xiaoping d'intervenir pour mettre fin aux mesures de répression.

Pékin proteste après l'adoption par le Parlement européen et par le Sénat des Etats-Unis de résolutions qui condamnent la répression au Tibet.

7-10.03.89 Conférence à Kuala Lumpur de délégués de 37 pays sur le problème des réfugiés en Indochine.

14-17.03.89 Le Premier ministre de Thailande en R.P. de Chine. Les deux pays définissent les principes jugés nécessaires à un règlement de la question cambodgienne : retrait de troupes vietnamiennes, et établissement d'un gouvernement de coalition nationale sous la direction du Prince Sihanouk, réduction de l'assistance militaire aux diverses factions cambodgiennes.

20.03.89 Ouverture de la 2ème session de la 7ème législature de la R.P. de Chine. Le Premier ministre M. Li Peng y présente le rapport 
sur le travail de son gouvernement. La politique de restructuration de l'économie continue mais le système socialiste ne sera pas abandonné.

La stratégie d'amélioration de l'environnement économique adoptée en septembre 1988 commence à obtenir des succès mais l'austérité durera encore plusieurs années en vue d'assurer la stabilité et l'unité.

Le montant des dettes extérieures doit être contrôlé.

L'enseignement bénéficiera d'une augmentation de $15.4 \%$ de son budget.

M. Li Peng souhaite des négociations avec le Dalai Lama à condition que ce dernier abandonne tout appui à l'idée d'indépendance du Tibet.

26.03.89 Premières élections à l'Assemblée populaire suprême du Laos (Parlement) depuis la formation de la R.P. démocratique en décembre 1975.

121 candidats, tous appuyés par le parti au pouvoir, contestent les 79 sièges à pourvoir.

26.03.89 Les élections législatives organisées en URSS sont les premières qui ont autorisé des candidatures multiples. Les mouvements réformateurs et nationalistes remportent un grand succès.

30.03.89 Le Ministre des Affaires Etrangères de Mongolie effectue en R.P. de Chine la première visite ministérielle mongole depuis l'établissement des relations diplomatiques. Trois accords sont conclus, sur l'établissement d'une commission de coopération économique, sur les échanges de visites entre citoyens des deux pays et sur le rétablissement d'un consulat mongol à Hohot (en Mongolie chinoise).

2-5.04.89 Visite officielle de M. Gorbatchev a Cuba. Le caractère fraternel et indestructible de l'amitié soviéto-cubaine est réaffirmé ainsi que le droit de chaque pays de définir sa conception du socialisme. Un traité d'amitié et de coopération est signé le 04.04.89. 
05.04.89 Les gouvernements vietnamien, laotien et cambodgien déclarent que toutes les troupes vietnamiennes seront retirées du Cambodge pour la fin du mois de septembre 1989. Ils demandent la fin de toute ingérence et de toute aide étrangère. La R.P. de Chine refuse la composition de la commission de contrôle international proposée par le Vietnam et souhaite un mécanisme placé sous la responsabilité du Secrétaire général de l'ONU. Le Prince Sihanouk rejette, le 6.4. la proposition des trois gouvernements.

07.04.89 M. Gorbatchev en visite officielle en Grande Bretagne met en garde les pays de l'OTAN contre une modernisation des armes nucléaires à courte portée en Europe.

12.04.89 Visite à Tokyo du Premier ministre de la R.P. de Chine; il est reçu par l'Empereur Akihito.

15.04.89 Mort de Hu Yaobang, ancien secrétaire général du P.C. de Chine, démis en janvier 1987 à la suite des manifestations estudiantines de décembre 1986.

Les étudiants manifestent en faveur de la libéralisation du régime et d'une réévaluation de l'action de Hu Yaobang.

Le 18.04, la foule tente de pénétrer dans le quartier des états majors du P.C.C. et du gouvernement.

Le 24.04, des grèves touchent 21 établissements d'enseignement supérieur a Pékin. Un appel est lancé aux ouvriers, commerçants et étudiants de toute la Chine de se joindre aux protestations contre l'indifférence officielle à l'égard de l'opinion publique.

18.04.89 Etablissement de relations diplomatiques entre la R.P. de Chine et Bahrein.

20.04.89 La R.P. de Chine rejette les conceptions de Taiwan qui nient le rôle du seul gouvernement légal de la Chine établi à Pékin. Son gouvernement n'a jamais renoncé aux moyens non pacifiques pour restaurer l'unité nationale parce que cette renonciation rendrait la réunification pacifique très difficile. 
23.04.89 Le Secrétaire général du P.C.C., M. Zhao Ziyang entame une visite en Corée du Nord.

La Chine réaffirme son appui à l'indépendance et à la réunification des deux parties de la péninsule coréenne et espère que des négociations entre la Corée du Nord et les EtatsUnis y mèneront.

24.04.89 Le Premier ministre de la R.P. du Kampuchea, M. Hun Sen annonce que des élections générales auront lieu en novembre 1989. Le 30.4, l'Assemblée nationale amende la Constitution du pays qui devient la République du Cambodge, un Etat neutre, pacifique et non aligné. Le bouddhisme devient religion nationale et la peine de mort est abolie.

25.04.89 Le Premier ministre japonais, M. Noboru Takeshita présente sa démission. Son successeur désigné le 2 mai est M. Sosuke Uno, qui, à son tour, sera mis en cause dès le 4 juin.

26.04.89 Un éditorial du "Quotidien du peuple" de Pékin exige de s'opposer aux troubles et aux activités qui sèment la division, créent le chaos et qui par une conspiration planifiée cherchent à renverser la direction du P.C.C. et le système socialiste.

Les manifestations non autorisées au préalable sont illégales et interdites.

La 38ème armée des forces populaire de libération est appelée à seconder la police pour maintenir l'ordre.

Dès le 22.4, des émeutes ont eu lieu à Xian, Changsha et au Sichuan.

Le Secrétaire du comité du P.C.C. de Shanghai, M. Jiang Zemin qui a suspendu l'édition d'un influent journal dès le 17.4, déclare le 26.4 que les grèves et manifestations illégales ont pu être évitées dans la grande municipalité.

Le 27.4, des milliers d'étudiants défilent sans autorisation dans le centre de Pékin; un entretien a lieu entre membres de l'administration gouvernementale et étudiants sous l'égide des organisations estudiantines officielles : la répression ne concerne que les agissements illégaux d'une minorité.

01.05.89 La délégation taiwanaise à la 22ème réunion annuelle de la Banque asiatique de développement arrive a Pékin. Elle est 
dirigée par le Ministre des finances du gouvernement de Taiwan.

02.05.89 Une brèche est ouverte dans la frontière entre la Hongrie et l'Autriche qui permet le passage de citoyens de l'Allemagne de l'Est vers l'Allemagne fédérale.

2-3.05.89 Rencontre à Jakarta entre le Prince Sihanouk et M. Hun Sen, Premier ministre cambodgien.

Du 8 au 10 mai ont lieu à Pékin des consultations entre vice ministres des Affaires étrangères de la R.P. de Chine et du Vietnam.

Le Vietnam refuse de lier le règlement cambodgien à l'établissement d'un gouvernement provisoire de coalition quadripartite et de geler l'importance des forces armées des 4 factions. Le Ministère chinois des Affaires étrangères affirme que le Vietnam cherche à légaliser le régime de Phnom Penh.

02.05.89 M. Yasser Arafat en France affirme sur les ondes de T.F. 1 que la Charte de l'OLP est devenue "caduque".

04.05.89 Le Secrétaire général du P.C.C. M. Zhao Ziyang s'adresse aux gouverneurs de la Banque asiatique de développement réunis à Pékin. Il déclare que les manifestations qui ont lieu en Chine ne prouvent pas une instabilité politique mais qu'elles expriment le souhait général de corriger les erreurs commises par le gouvernement et le P.C.C. Il estime que les exigences des étudiants doivent être rencontrées par des moyens démocratiques et légaux.

Dès le 3 mai, le gouvernement de la R.P. de Chine a fait savoir qu'il est disposé à un dialogue avec les étudiants mais pas à des négociations et il refuse qu'elles aient lieu sur un plan d'égalité entre ses représentants et les étudiants.

Le 3 mai également, une déclaration d'intellectuels chinois exige que les libertés garanties par la Constitution soient respectées et que leur exercice ne soit pas considéré comme un complot planifié.

A l'occasion du 70ème anniversaire du mouvement du 4 mai 1919, M. Zhao Ziyang a, dans un discours prononcé le 3 mai, lié l'esprit de 1919 aux besoins de la Chine depuis 1978. Le succès 
des réformes en cours est fondé sur l'engagement patriotique, démocratique et scientifique mais la modernisation socialiste exige un environnement social et politique stable.

La progression de la démocratie doit se faire de manière ordonnée et respecter le droit, donc la direction du P.C.C. qui lui-même doit être délivré de la corruption.

Plus de 200.000 étudiants défilent à Pékin le 4.5 sans confrontation, avec la participation de délégués des principales universités du pays et de journalistes qui veulent que les événements soient librement et honnêtement rapportés.

04.05.89 Deux dirigeants du mouvement indépendantiste de Nouvelle Calédonie sont tués par un extrémiste Canaque.

05.05.89 Le Premier ministre du Cambodge, M. Hun Sen propose à son homologue thailandais, à Bangkok, que la Thailande assume un rôle spécial de contrôle sur le retrait des forces vietnamiennes du Cambodge.

05.05.89 Le Président du Parlement iranien appelle les Palestiniens a "tuer des Américains, des Britanniques et des Français" en réponse à la mort de Palestiniens dans les territoires occupés par Israël.

8-10.05.89 Des conversations ont lieu à Pékin entre le Ministre des Affaires Etrangères de la R.P. de Chine et le vice ministre vietnamien des Affaires étrangères.

09.05.89 Le gouvernement de la Corée du Sud entame une sévère répression du mouvement radical de gauche, responsable de manifestations violentes depuis plusieurs mois.

11.05.89 M. Gorbatchev reçoit le Secrétaire d'Etat J. Baker; il annonce le retrait unilatéral de 500 ogives nucléaires tactiques et formule de nouvelles propositions de réduction des forces conventionnelles en Europe.

12.05.89 Le Président des Etats-Unis demande à l'URSS de prouver sa bonne volonté en "déchirant le rideau de fer". 
13.05.89500 étudiants entament une grève de la faim devant le monument aux héros de la révolution, place Tiananmen à Pékin. M. Zhao Ziyang demande aux citoyens chinois de ne rien faire qui puisse gêner le prochain sommet sino-soviétique. Le 15 mai, entre 500.000 et 800.000 manifestants sont massés sur la place Tiananmen : étudiants, chercheurs, enseignants, journalistes, cadres, fonctionnaires et d'importants contingents d'ouvriers.

15 au 18.05.89 Le protocole d'accueil de M. Gorbatchev à Pékin est modifié en raison des manifestants massés au coeur de la ville. Le $16 \mathrm{mai}$, M. Gorbatchev rencontre successivement MM. Deng Xiao Ping, Zhao Ziyang et Li Peng.

M. Zhao Ziyang se réjouit de ce que la normalisation ait été annoncée le matin même et révèle le rôle préeminent dévolu à $M$. Deng par une décision restée secrète.

Les relations entre les deux pays sont placées sur l'égide de quatre principes : indépendance, totale égalité, respect mutuel et non ingérence dans les affaires internes.

Les facteurs négatifs qui ont perturbé les relations entre la R.P. de Chine et l'URSS ont été surmontés; les rapports entrent dans une nouvelle étape.

L'URSS va retirer $75 \%$ de ses troupes stationnées en Mongolie et souhaite transformer les régions frontalières entre la Chine et l'URSS en zone de paix et de bon voisinage.

Aucune mention n'est faite d'un accord sur le Cambodge.

18.05.89 Le Premier ministre de R.P. de Chine, M. Li Peng en compagnie d'autres dirigeants reçoit 12 représentants des grévistes de la faim.

Les étudiants veulent que l'éditorial du 26 avril soit remanié et que soit reconnue la signification de leur mouvement.

Le Premier Ministre déclare que certaines aspirations des étudiants sont patriotiques et que quelques-unes font l'objet d'examens mais la capitale est en état d'anarchie et le chaos s'étend au réseau des chemins de fer.

Il exige une dernière fois l'arrêt de la grève.

19.05.89 Le Secrétaire général du P.C.C. Zhao Ziyang se rend auprès des étudiants grévistes et les supplie d'arrêter leur mouvement. 
Une pétition envoyée par l'Université de Shenzhen, demande la démission de M. Deng Xiaoping et la réunion du Comité central du P.C.C. car le "parti doit refléter, respecter, s'accorder avec la volonté du peuple qu'il représente et non être manipulé par une éminence grise".

A une réunion de cadres du P.C., du gouvernement et de l'armée convoquée par le C.C. et le Conseil d'Etat, M. Li Peng déclare que des mesures fermes doivent être prises car "une poignée de gens utilisent les grévistes de la faim comme otages pour contraindre le P.C. et le gouvernement à se soumettre à leurs exigences politiques", M. Zhao Ziyang n'assiste pas à cette réunion et sera en conséquence accusé de rompre l'unité du P.C. et de violer le centralisme démocratique.

Une polarisation s'opere au sein de la direction entre ceux qui, avec M. Li Peng, considerent les manifestations comme un soulèvement à réprimer et ceux qui comme $M$. Zhao Ziyang recherchent des accommodements afin d'accélérer la démocratisation et les réformes politiques demandées par les étudiants.

20.05.89 Le Premier ministre M. Li Peng instaure la loi martiale dans certains quartiers de Pékin et fait appel a l'armée pour rétablir l'ordre.

Les manifestations continuent.

21.05.89 M. Yu Kuo Hwa qui a démissioné de son poste de Premier ministre à Taiwan est remplacé par le Secrétaire général du K.M.T., M. Lee Huan.

21.05.89 Après les élections qui ont eu lieu le $17 \mathrm{mai}$, une grande assemblée des tribus est organisée en Afghanistan.

M. Najibullah y demande la réhabilitation politique des groupes d'opposition armée auquel il promet des commandements militaires et déclare être disposé à accorder l'autonomie a certaines parties du territoire. Le 21.5, l'état d'urgence est prolongé pour 6 mois.

24.05.89 Le Président de la R.P. de Chine, M. Yang Shangkun accuse le Secrétaire général du P.C. d'avoir refusé d'appliquer les 
instructions et d'avoir parlé inconsidérément avec $M$. Gorbatchev le 16.5 .

Le Président du Comité permanent de l'Assemblée populaire nationale (Parlement), M. Wan Li écourte sa visite aux EtatsUnis; depuis Shanghai, il déclare que la loi martiale a été décrétée conformément à la Constitution.

Le $26.5, \mathrm{M}$. Peng $\mathrm{Zhen}$, ancien membre du bureau politique du P.C.C. et ancien président du Comité permanent de l'Assemblée nationale, condamne les méthodes utilisées par les étudiants; la promotion de la libéralisation bourgeoise est inconstitutionnelle et nuit aux intérêts de la population et à la réalisation du programme du P.C.C.

25.05.89 M. Gorbatchev est élu chef de l'Etat en URSS.

26.05 aucun des réformateurs les plus connus n'est élu parmi les 542 membres des assemblées législatives permanentes du Soviet Suprême.

29.05 M. B. Eltsine obtient d'y siéger grâce à la démission d'un des représentants élus.

$30.05 \mathrm{M}$. Gorbatchev s'engage à renforcer le rôle du Parlement et augmente les crédits destinés à l'armée.

29-30.05.89 Le sommet de l'OTAN entérine les propositions de désarmement du Président des Etats-Unis.

Un accord sur les forces conventionnelles est un préalable à des pourparlers sur la réduction des missiles nucléaires à courte portée en Europe.

29.05.89 Les manifestations continuent à Pékin. Les étudiants de l'Institut Central des Beaux Arts érigent une statue de la "déesse de la démocratie" sur la place Tiananmen.

31.05.89 Des ouvriers et des paysans défilent dans les faubourgs de Pékin en faveur de la loi martiale.

L'armée est déployée dans les faubourgs et à des endroits stratégiques. La population de Pékin érige des barricades pour empêcher la pénétration de l'armée dans la ville et s'en prend à certains militaires. 
30.05 au 23.06.89 A la conférence sur les droits de l'homme réunie à Paris, la Roumanie et la Bulgarie sont accusées de ne pas respecter leurs engagements souscrits en 1975 à Helsinki.

03.06.89 Mort de l'imam Khomeiny, remplacé comme guide de la révolution iranienne par le chef de l'Etat, M. Ali Khamenei.

03.06.89 Des troubles ethniques perturbent l'Ouzbekistan et le 17.06 le Kazakstan.

04.06.89 M. Pol Pot, ancien Premier Ministre du Kampuchea démocratique de 1976 à 1979, démissionne de toutes ses fonctions au sein des Khmers Rouges.

04.06.89 L'armée chinoise intervient en force à Pékin et rétablit l'ordre après l'obstruction mise par la population à la progression des troupes et des attaques perpétrées contre les soldats de l'Armée populaire. Les troubles créés par une petite minorité sont qualifiés depuis le 03.06 d'"horrible complot contrerévolutionnaire d'avocats de la libéralisation bourgeoise en collusion avec des forces étrangères hostiles".

Les citoyens sont invités à dénoncer ceux qui répandent des rumeurs ou commettent des actes criminels. A Hong Kong, la population témoigne son soutien au mouvement par des défilés massifs et la presse, y compris celle favorable à la R.P. exprime l'horreur que provoque la brutale répression utilisée.

05.06.89 Taiwan place ses forces armées en alerte et le Conseil législatif y condamne le massacre de Pékin.

05.06.89 Les Etats-Unis suspendent toute vente d'armes à la R.P. de Chine, les échanges gouvernementaux et proposent aux organisations internationales de postposer l'octroi de crédits.

La CEE déplore la répression brutale de la population de Pékin et avertit que la coopération avec la R.P. de Chine en souffrira. Le gouvernement français gèle les relations politiques avec la R.P. de Chine et le Président Mitterrand demande la clémence pour les personnes impliquées dans les événements. 
La République fédérale d'Allemagne gèle tout nouvel engagement à l'égard de la R.P. de Chine en raison des violations commises aux Droits de l'homme.

05.06.89 L'Australie annule la visite que devait faire en R.P. de Chine son Premier ministre B. Hawke. Le gouvernement étend la durée des permis de séjour accordés aux citoyens chinois en Australie.

La Suisse interdit toute vente d'armes à la R.P. de Chine.

Les gouvernements de Malaysie, des Philippines, de Corée du Sud, du Pakistan, du Bangladesh et de l'Inde considèrent que leurs relations avec la R.P. de Chine ne sont pas affectées par les événements.

09.06.89 M. Deng Xaoping à Pékin félicite les militaires qui ont écrasé le complot contre-révolutionnaire.

Il déclare que la "ligne, les principes et la politique appliqués depuis décembre 1978 sont corrects", qu'il faut respecter les quatre principes cardinaux (voie socialiste, dictature démocratique du peuple, rôle dirigeant du P.C.C., respect du marxisme léninisme et de la pensée de Mao Zedong) et réaliser les réformes et l'ouverture du pays.

Les autorités chinoises considèrent que les événements constituent une affaire intérieure dans laquelle aucune ingérence étrangère n'est permise. Un exemple d'ingérence est fourni par l'asile donné par l'ambassade des Etats-Unis à Pékin à M. et Mme Fang Lizhi.

11.06.89 Les élections provinciales en Nouvelle Calédonie confèrent au F.L.N.K.S., 19 des 54 sièges au Congrès du territoire et la majorité absolue dans deux des trois assemblées provinciales.

14.06.89 La conférence internationale sur les réfugiés indochinois réunie à Genève adopte un plan de rapatriement volontaire des "boat people" vietnamiens et laotiens mais accepte à terme le principe du rapatriement forcé. Cette conférence discute aussi du sort des quelques 300.000 réfugiés cambodgiens et laotiens qui vivent dans des camps en Thailande. 
20 au 23.06.89 M. H. Rafsandjani, Président du Parlement iranien conclut à Moscou plusieurs accords bilatéraux. L'Agence Tass souligne l'identité de nombreuses positions entre MM. Gorbatchev et Rafsandjani.

23-24.06.89 4ème session plénière du 13ème C.C. du P.C. chinois. M. Zhao Ziyang est démis de tous ses postes mais reste membre du P.C.C. M. Jiang Zemin, secrétaire du comité du P.C.C. de Shanghai est promu Secrétaire général du P.C.C.

Trois membres du secrétariat du C.C. sont relevés de leurs fonctions parmi lesquels M. Hu Qili, qui était également membre du comité permanent du bureau politique.

1-2.07.89 Le 13ème festival mondial de la jeunesse et des étudiants s'ouvre à Pyongyang, capitale de la Corée du Nord. 180 pays et organisations y sont représentés.

01.07.89 A l'occasion du 68dme anniversaire du P.C. chinois, les autorités rappellent les priorités : le respect du marxisme et de la pensée de Mao Zedong, l'opposition à la libéralisation bourgeoise, l'élimination de la rébellion contrerévolutionnaire, l'étude des erreurs de travail du P.C., le renforcement de l'effort politique et idéologique.

Les réformes doivent s'opérer dans le cadre renforcé de la planification et de la propriété publique : il s'agit de perfectionner le système socialiste et non de changer de système.

3-4.07.89 22æme réunion annuelle des Ministres des Affaires étrangères de l'ASEAN à Brunéi.

14.07.89 Le Sénat des Etats-Unis adopte des sanctions à l'égard de la R.P. de Chine.

14.07.89 L'état d'urgence est imposé à Bougainville afin de lutter contre les troubles qui cherchent a faire sécession de PapouasieNouvelle Guinée.

15.07.89 La déclaration adoptée au 15ìme sommet des sept pays les plus industrialisés invite la R.P. de Chine à cesser de persécuter ceux 
qui n'ont que clamé leur droit à la démocratie et à la liberté. Il y est décidé de postposer tout nouveau prêt de la Banque Mondiale à la R.P. de Chine et de demander au gouvernement de Pékin de restaurer la confiance à Hong Kong.

16-17.07.89 Visite au Pakistan du Premier ministre indien, M. R. Gandhi.

20.07.89 Des citoyens chinois recherchés comme contrerévolutionnaires par les autorités de Pékin, réfugiés à l'étranger, annoncent la création d'un front démocratique destiné à renverser le régime de la R.P. de Chine par des moyens pacifiques.

21.07.89 Le Conseil militaire (SLORC) au pouvoir en Birmanie (dont le nom officiel est Myanmar) place sous résidence surveillée pour un an, les plus importants dirigeants de l'opposition.

23.07.89 Le parti libéral démocrate n'obtient que 109 des 252 sièges a pourvoir aux élections pour la Chambre haute du Parlement du Japon.

Le Premier ministre M. Uno présente sa démission et le 8.8, M. Toshiki Kaifu devient président du parti libéral démocrate et le 9.08, Premier Ministre.

24.07.89 Le Ministre des Affaires Etrangères de la R.P. de Chine, M. Qian Qichen entreprend une tournée en Afrique : Botswana, Zimbabwe, Angola, Zambie, Mozambique et Lesotho.

24-31.07.89 Le parti unique au pouvoir au Mozambique depuis 1975, le FRELIMO, opte pour la libéralisation de l'économie et l'ouverture à l'Occident.

26.07.89 M. Fidel Castro dénonce la libéralisation en cours en URSS, en Hongrie et en Pologne et affirme que "la révolution cubaine continuera à résister".

01.08.89 Un plan de libéralisation des prix agro-alimentaires entre en vigueur en Pologne. 
07.08.89 M. David Lange, Premier ministre de Nouvelle Zélande depuis 1984, démissionne.

M. Geoffrey Palmer lui succède le 8.08.

16.08.89 "Solidarité" et les partis paysan et démocrate souhaitent un gouvernement de coalition en Pologne, avec la participation des communistes.

19.08. M. Tadeuz Mazowiecki devient Premier ministre. Le gouvernement polonais est formé le 12.09; il comprend quatre ministres communistes aux portefeuilles de l'intérieur, de la défense, des transports et du commerce extérieur.

12-18.08.89 Les relations sont restaurées entre le Parti révolutionnaire du peuple laotien (le P.C.) et le P.C. chinois lors de la visite du Vice Ministre des Affaires Etrangères du Laos en R.P. de Chine.

16.08.89 Le Ministre des Affaires Etrangères de la R.P. de Chine condamne la réunion internationale organisée à la nouvelle Delhi a propos du Tibet et de la paix en Asie du Sud-Est, comme une grossière ingérence dans les affaires intérieures chinoises.

19.08.89 A la suite du rétablissement des liens diplomatiques entre Taiwan et la Grenade, la R.P. de Chine suspend ses relations avec la Grenade noués en 1985.

21.08.89 Le 21ème anniversaire de l'intervention des troupes du Pacte de Varsovie en Tchécoslovaquie est commémoré par des manifestants à Prague.

22.08.89 La Turquie instaure un visa d'entrée obligatoire afin d'arrêter le flux de réfugiés bulgares d'origine turque.

22.08.89 Le Ministre des Affaires Etrangères de la R.P. de Chine rappelle, a l'occasion de la proposition d'établir une base militaire américaine à Singapour, que le gouvernement de Pékin s'oppose à toutes les bases militaires étrangères. 
23.08.89 Une chaîne humaine de $560 \mathrm{~km}$ est formée à travers la Lituanie, la Lettonie et l'Estonie pour rappeler la conclusion du pacte germano-soviétique du 23.8.1939.

30.8.89 La conférence internationale sur le Cambodge qui réunit à Paris les chefs des quatre factions cambodgiennes, les ministres des Affaires étrangères des cinq membres permanents du Conseil de Sécurité, des représentants des principales nations d'Asie ainsi que de l'Australie, du Canada et le Secrétaire général de l'ONU, suspend ses travaux; elle organise des commissions et définit leurs objectifs. Selon le Ministre des Affaires Etrangères de la R.P. de Chine, le Prince Sihanouk est la seule personne acceptée par la Communauté internationale qui soit capable de rassembler les quatre forces politiques du Cambodge.

30.08.89 "Amnesty International" remet à l'ONU et a la R.P. de Chine un rapport qui reprend les témoignages sur la répression du "printemps de Pékin".

31.08.89 Le gouvernement de la R.P. de Chine rejette catégoriquement la résolution du Comité des Nations Unis sur les droits de l'homme comme ingérence dans les affaires intérieures, tentative de pression et violation de la Charte des NationsUnis et des normes du droit international.

01.09.89 Le général Noriega installe un nouveau Président de la République à Panama; Washington annonce de nouvelles sanctions économiques. Le ministère des Affaires étrangères de R.P. de Chine critique l'engagement américain au Panama : "tentative de forcer le gouvernement Noriega à plier devant l'opposition".

01.09.89 Le Premier ministre japonais M. Kaifu à Washington rencontre le Président G. Bush.

Les entretiens portent sur les problèmes de sécurité et sur les relations commerciales entre les deux pays.

4-7.09.89 Au 9ème sommet des non alignés réuni à Belgrade, la Yougoslavie qui assume la direction du mouvement fait 
adopter une ligne qui n'est plus axée sur l'opposition à l'impérialisme.

05.09.89 Le Président des Etats-Unis présente un vaste plan de lutte contre la drogue.

7-12.09.89 La visite en URSS du Président de la République d'Indonésie, M. Suharto marque la normalisation des relations entre les deux pays après les événements de septembre 1965.

Conclusion d'accords de coopération économique et d'échanges commerciaux.

11.09.89 A l'occasion de la fête nationale de la Corée du Sud, le Président Roh Tae Woo propose un plan de réunification et une rencontre avec le Président de la Corée du Nord, M. Kim Il sung.

17.09.89 Une délégation parlementaire japonaise en R.P. de Chine. Le Japon demande la levée de la loi martiale a Pékin et une déclaration d'engagement dans une politique de réformes et d'ouverture.

18.09.89 Un accord est signé a Colombo entre les Ministres des Affaires étrangères de l'Inde et de Sri Lanka à propos de l'évacuation pour le 31.12.1989 des forces armées indiennes de l'île.

18.09.89 La Hongrie rétablit des relations diplomatiques avec Israël, rompues en juin 1967.

22-23.09.89 L'URSS renonce à lier la conclusion d'un accord sur la limitation des armements stratégiques à l'abandon par les Etats-Unis du projet d'initiative de défense stratégique (IDS ou"guerre des étoiles").

24.09.89 Fondation à Paris de la Fédération pour la démocratie en Chine par des dissidents chinois réfugiés à l'étranger.

Les autorités de la R.P. de Chine accusent la France de soutenir des criminels de droit commun. 
25-26.09.89 Le Conseil de coopération arabe, établi en février 1989 entre le Yemen, la Jordanie, l'Irak et l'Egypte et réuni au Yemen, adopte des accords économiques qui cherchent à fonder un marché commun sur le modèle de la C.E.E.

25.09.89 Une manifestation importante à Leipzig en Allemagne de l'Est exige des réformes et la reconnaissance du "Nouveau Forum".

26.09.89 M. Chevardnadze, le Ministre soviétique des Affaires étrangères accepte des négociations avec les Etats-Unis sur la réduction des stocks d'armes chimiques mais il en demande l'élimination totale.

26.09.89 Le gouvernement du Vietnam déclare que toutes les forces armées vietnamiennes ont été retirées du Cambodge.

Le Prince Sihanouk déclare que ce retrait est "factice".

Pour la R.P. de Chine, il faut que Hanoi prouve sa sincérité en acceptant une vérification du retrait par l'ONU.

26.09.89 La CEE est chargée de coordonner l'aide des pays occidentaux à la Pologne et a la Hongrie et met à l'étude le soutien financier à accorder à la démocratisation de l'Europe orientale.

27.09.89 Le "Quotidien du peuple" réaffirme l'engagement de la Chine dans la politique de réunification pacifique basée sur le principe de "un pays, deux systèmes". Le gouvernement de la R.P. de Chine regrette que Taiwan continue à rechercher la double reconnaissance.

27-29.09.89 Réunion à Londres du groupe de liaison sino-britannique a propos de Hong Kong dont les travaux ont été suspendus depuis la répression du "Printemps de Pékin".

La R.P. de Chine critique l'appui implicite de la Grande Bretagne à une minorité qui a voulu utiliser Hong Kong pour saboter le gouvernement de Pékin.

28.09.89 L'ancien Président des Philippines, renversé en 1986, M. F. Marcos, meurt à Hawai. 
29.09.89 A l'occasion du 40ème anniversaire de la fondation de la R.P. de Chine, le Secrétaire général du P.C., M. Jiang Zemin déclare que la rébellion a été une expression de la lutte des classes et que les réformes doivent être conformes à l'orthodoxie marxiste, c'est-à-dire qu'elles rejettent la libéralisation bourgeoise, l'occidentalisation, l'intégration de la Chine dans le système capitaliste mondial.

4-6.10.89 Visite en R.P. de Chine de M. Kim Il-sung, Président de la Corée du Nord. M. Deng Xiaoping lui déclare que la R.P. de Chine n'établira que des relations inofficielles avec la Corée du Sud.

05.10.89 Le prix Nobel de la paix est attribué au Dalai Lama qui vit en exil depuis 1959.

La R.P. de Chine exprime son indignation extrême pour cette ingérence dans ses affaires intérieures et cette tentative d'inculquer les idées occidentales de liberté et de droits de l'homme.

05.10.89 Le chef de l'OLP, M. Yasser Arafat est reçu en R.P. de Chine.

Le Premier ministre, M. Li Peng propose cinq points pour un règlement politique au Moyen Orient, basé sur une conférence internationale sous l'égide des Nations Unies. Il invite Israël et la Palestine à se reconnaître.

5-10.10.89 Le Président du Conseil des ministres du Laos qui est aussi Secrétaire général du parti révolutionnaire du peuple laotien, signe en R.P. de Chine des acords sur les frontières sinolaotiennes, un accord consulaire et un accord de coopération culturelle.

06.10.89 Le Pape Jean Paul II se rend en Corée du Sud, en Indonésie (à Timor) et à l'Ile Maurice.

6-7.10.89M. Gorbatchev en République démocratique allemande encourage aux réformes.

Le 18.10, M. E. Honecker, Secrétaire général du parti socialiste unifié depuis 1971, est remplacé par M. Egor Krentz qui exprime la volonté du "changement dans la continuité". 
07.10.89 Le parti socialiste ouvrier de Hongrie (P.C. au pouvoir) devient le parti socialiste hongrois et abandonne les principes du centralisme démocratique et de la dictature du prolétariat. 20.10, la Constitution est modifiée, les élections seront libres et multipartites.

23.10, La IVème république est proclamée en Hongrie.

09.10.89 Taiwan et le Liberia rétablissent leurs relations diplomatiques. La R.P. de Chine suspend ses relations avec le Liberia et impute l'évolution à la diplomatie "élastique" et "du dollar" de Taiwan.

11-18.10.89 Le rétablissement des relations diplomatiques entre Taiwan et Belize entraine la suspension par la R.P. de Chine de ses relations diplomatiques avec Belize.

11-18.10.89 Le Vice Premier ministre de la R.P. de Chine effectue une visite en Inde.

16.10.89 Un nouveau gouvernement est formé a Tuvalu, après les élections organisées le 27.09 .

18.10.89 Le Gouvernement de Bonn décide de libérer les prêts d'aide au développement destiné à la construction du métro de Shanghai et gelé après le 04.06.89.

15-20.10.89 Le Président de la Corée du Sud, M. Roh Tae Woo est reçu aux Etats-Unis.

Les troupes américaines resteront stationnées en Corée du Sud tant que subsistera la menace du Nord; le gouvernement de Séoul s'efforce de prendre en charge une part plus importante du fardeau de la Défense et ouvrira davantage le marché aux produits américains.

18-24.10.89 La réunion à Kuala Lumpur des chefs de gouvernement du Commonwealth réintègre le Pakistan.

19.10.89 La Finlande accorde un prêt à la R.P. de Chine pour financer l'équipement d'une usine à papier; il s'agit de la première offre 
d'aide occidentale à Pékin depuis les événements de mai et juin 1989.

22.10.89 La guerilla des Khmers Rouges capture la ville de Pailin moins d'un mois après le retrait des forces vietnamiennes du Cambodge.

30.10.89 Le couvre feu est décrété à Phnom Penh.

23.10.89 M. Chevardnadze critique, devant le Soviet Suprême, la direction précédente de l'URSS qui a décidé l'intervention en Afghanistan.

24.10.89 L'Ambassadeur de R.P. de Chine a Manille proteste formellement auprès du gouvernement des Philippines pour la visite "inofficielle" à Taiwan du Secrétaire des Affaires étrangères philippin les 21-23.10.

27.10.89 Signature d'un traité consulaire entre la R.P. de Chine et l'Irak.

25-31.10.89 L'Assemblée nationale populaire de la R.P. de Chine adopte une nouvelle législation sur les manifestations. La police pourra faire usage d'armes pour disperser des réunions illégales. Il est interdit aux citoyens de manifester en dehors de la ville dans laquelle ils sont enregistrés comme résidents.

Depuis le 15.09, les citoyens chinois sont obligés de porter leur carte d'identité en permanence.

28.10.89 Une énorme manifestation célébre à Prague l'anniversaire de la République tchécoslovaque établie en 1918.

01.11.89 La Corée du Sud et la Pologne établissent des relations diplomatiques. La Corée du Nord rappelle son ambassadeur de Varsovie, comme elle l'avait fait précédemment à l'égard de la Hongrie lorsque des liens ont été établis entre Budapest et Séoul.

01.11.89 L'Assemblée générale de l'ONU adopte une résolution sur l'Afghanistan. Elle demande que les parties respectent la lettre et l'esprit des accords du 14.04.88. 
05.11.89 Le Conseil de coopération économique de l'Asie-Pacifique est créé par 12 pays réunis en conférence a Canberra : les membres de l'ASEAN, les Etats-Unis, le Canada, le Japon, la Corée du Sud, l'Australie et la Nouvelle Zélande.

05.11.89 M. Mladenov, Ministre des Affaires Etrangères de Bulgarie signe en R.P. de Chine trois accords de coopération.

Le 10.11, M. Mladenov remplace à la direction du P.C. M. Todor Jivkov au pouvoir en Bulgarie depuis 1954.

6-9.11.89 5ème session plénière du 13ème C.C. du P.C. chinois. Deng Xiaoping démissionne de la présidence de la commission militaire centrale du parti. M. Jiang Zemin lui succède et le Président de la République, M. Yang Shang-Kun devient vice président de la Commission militaire centrale.

L'austérité doit continuer pendant deux ou trois ans afin de lutter contre la surchauffe de l'économie et construire la base d'une réforme accélérée ultérieure.

Une décision sur "l'amélioration de l'environnement économique, la rectification de l'ordre économique et l'approfondissement de la réforme" est adoptée.

8-9.10.89 Les manifestations quotidiennes dans les grandes villes provoquent la démission du gouvernement de M. E. Krentz en Allemagne de l'Est.

Les instances dirigeantes du parti au pouvoir (Parti socialiste unifié) sont renouvelées.

Le chef du parti de Dresde, favorable à des réformes, M. H. Modrow est chargé de former un nouveau gouvernement.

Le 9.11, le mur qui divise Berlin depuis 1961 est ouvert.

Le 24.12, la liberté de circulation est totale entre les deux Allemagne.

12.11.89 Une délégation du Soviet Suprême de l'Union Soviétique conduite par M. Yakovlev mène des entretiens au Japon. Il fait état d'une "troisième voie" pour résoudre le différend territorial entre l'URSS et le Japon. 
13.11.89 Au Sri Lanka, une opération est mence contre le J.V.P., le mouvement cinghalais de gauche, responsable des troubles. Ses dirigeants sont arrêtés ou tués.

16.11.89 Le Premier ministre M. Li Peng en visite au Pakistan. La R.P. de Chine fournira une centrale nucléaire dont la construction se fera sous le contrôle de l'organisation internationale de l'usage pacifique de l'énergie nucléaire.

17.11.89 La répression d'une manifestation d'étudiants à Prague mobilise la population contre le régime.

M. A. Dubcek est ovationné par la foule le 24.11, le bureau politique du P.C. démissionne, des négociations ont lieu avec des représentants des mouvements indépendants regroupés dans le Forum civique et le 29.11, le rôle dirigeant du P.C. est aboli.

18.11.89 M. Roh Tae Woo, Président de la Corée du Sud mène une tournée en Europe : en R.F.A., en Hongrie avec laquelle des relations diplomatiques ont été nouées en février 1989, en Grande Bretagne et en France.

22.11.89 Le Premier ministre de la R.P. de Chine signe au Népal un accord qui prévoit la construction de nouvelles routes et l'entretien de celles déjà construites avec l'aide la Chine.

22-26.11.89 Elections générales en Inde pour renouveler la Chambre des députés, dans une atmosphère de grande tension. Le parti du Congrès remporte 192 sièges, le Janata et le Front national 144 sieges, le parti hindouiste 86 et la gauche 51 , sur un total de 525 sieges à pourvoir.

24.11.89 M. N. Ceaucescu est rélu à l'unanimité Secrétaire général du P.C. roumain qu'il dirige depuis 1965 . Il exclut toute remise en cause du socialisme et condamne les déviations d'autres pays socialistes.

Le P.C. de Chine félicite chaleureusement M. N. Ceaucescu.

24.11.89 Un accord de commerce et de coopération est conclu entre la C.E.E. et l'URSS. 
29-30.11.89 M. Gorbatchev en visite officielle en Italie évoque l'avenir d'une Europe réconciliée dans le respect des équilibres actuels. Il suggère la réunion en 1990 des 35 membres de la conférence sur la sécurité et la coopération en Europe.

30.11.89 M. Chevardnadze, Ministre des Affaires Etrangères de l'URSS a des entretiens a Rome avec l'ancien Roi d'Afghanistan, Zaher Shah.

01.12.89 M. Gorbatchev est reçu par le Pape au Vatican. Il s'engage au respect de la liberté religieuse dans son pays. Le prochain rétablissement des relations diplomatiques entre le Vatican et l'URSS est décidé.

01.12.89 Une tentative de coup militaire contre la Présidente C. Aquino échoue aux Philippines, grâce au soutien de l'aviation américaine.

01.12.89 Un projet commun de Constitution est adopté pour le Yemen réunifié par les dirigeants de la République Arabe du Yemen et ceux de la république populaire du Yemen. Ce projet prévoit le pluripartisme politique. Les 23 et 26 décembre, les deux gouvernements yeménites grâcient tous les prisonniers politiques de leur pays.

02.12.89 Le P.C. malais signe des traités de paix séparés avec les gouvernements de Malaysie et le commandement des opérations de sécurité interne de Thailande, mettant ainsi fin à 41 années d'insurrection armée.

02.12.89 Aux élections législatives organisées à Taiwan, le K.M.T. au pouvoir remporte 72 des 101 sièges à pourvoir.

Le parti démocratique progressiste obtient 21 sièges.

Le 01.12, le chef d'Etat major le Général Hau Pei-tsun devient Ministre de la défense nationale.

2-3.12.89 MM. Bush et Gorbatchev, au cours de leur premier sommet annoncent, à Malte, l'ouverture d'une "ère nouvelle" dans les relations internationales et décident d'accélérer les négociations sur le désarmement. 
03.12.89 Le C.C. et le bureau politique du parti communiste d'Allemangne de l'Est annoncent leur dissolution, M. E. Krentz démissionne le 6.12.

Le 9.12, le parti abolit son rôle dirigeant, accepte le pluralisme politique et élit un nouveau président M. Gregor Gysi.

Le Premier ministre de la R.P. de Chine se déclare préoccupé par la rapidité de l'évolution. Elle n'influencera cependant pas la détermination chinoise à conserver son système socialiste.

04.12.89 Les dirigeants des pays membres de l'OTAN expriment leur volonté de préserver les frontières et les organisations politicomilitaires qui existent en Europe. Ceux du Pacte de Varsovie en font de même le 6.12 et condamnent l'intervention de 1968 en Tchécoslovaquie.

05.12.89 Echange de notes entre le Japon et la R.P. de Chine à propos de l'octroi d'un prêt japonais promis en 1988 donc antéricur aux décisions de gel de la coopération annoncées le 1.8 .89 par le Ministre des Affaires Etrangères du Japon.

Une délégation parlementaire japonaise s'est déjà rendue en R.P. de Chine en septembre 1989 et y a souhaité la levée de la loi martiale.

6-8.12.89 Un accord entre le Premier ministre de l'Inde et le Ministre des Affaires Etrangères du Sri Lanka reporte au 31.01.1990 le retrait des forces indiennes de l'île.

07.12.89 L'Irak annonce le lancement réussi de sa première fusée capable de placer un satellite sur orbite spaciale.

9-10.12.89 Deux émissaires du Président Bush, parmi lesquels M. Brent Scowcroft, assistant pour la sécurité nationale, s'entretiennent à Pékin avec les autorités de la R.P. de Chine.

$M$. Deng Xiaoping espère que cette visite conduira à la solution des différends et à un nouvel élan dans les relations bilatérales.

10.12.89 Sous la pression des manifestations un gouvernement d'entente nationale est constitué en Tchécoslovaquie. Il promet des élections libres dans les six mois et une économie de marché. 
28.12., M. A. Dubcek est élu président du Parlement et le 29.12, M. V. Havel est élu président de la République.

11.12.89 Des manifestants réclament l'accélération des réformes en Bulgarie; le P.C. promet des élections libres en mai 1990 et abolit son rôle dirigeant.

11.12.89 Accord entre l'Indonésie et l'Australie sur l'exploitation en commun des réserves de pétrole et de gaz dans la région des frontières maritimes contestées dans la fosse de Timor.

11-14.12.89 M. Kaysone Phomvihane, premier ministre du Laos effectue la premiere visite d'un dirigeant laotien en France depuis 1975.

12.12.89 Le rapatriement forcé de 51 réfugiés vietnamiens d'Hong Kong conformément à un accord entre la Grande Bretagne et le Vietnam est vivement critiqué dans le monde.

12.12.89 Le débat demandé par M. Sakharov au Congrès des députés de l'Union soviétique sur l'abolition du rôle dirigeant au P.C. est refusé par M. Gorbatchev et une large majorité des députés.

14.12.89 Mort en URSS d'Andrei Sakharov, Prix Nobel de la paix 1975.

16.12.89 Des discussions ont lieu a Hong Kong entre "hommes d'affaires" de la R.P. de Chine et de Taiwan.

18-21.12.89 Réunion à Muscat (Oman) du Conseil supérieur de coopération du Golfe Persique.

Les chefs d'Etat des six pays membres (Bahrain, Koweit, Oman, Qatar, Arabic Saoudite, Emirats Arabes Unis) invitent Oman à prendre une initiative diplomatique pour contribuer à un réglement de paix entre l'Iran et l'Irak.

18.12.89 Une nouvelle organisation, l'Union démocratique mongole, manifeste à Ulan Bator, pour être reconnue par le régime du parti communiste au pouvoir en Mongolie. 
20.12.89 Le P.C. lituanien proclame son autonomie par rapport au P.C. de l'URSS et adopte un programme distinct. Ces décisions sont critiquées par M. Gorbatchev le 25.12.

20.12.89 Les Etats-Unis lancent au Panama une opération militaire pour restaurer le processus démocratique et capturer le Général Noriega inculpé de trafic de drogue.

L'URSS et la R.P. de Chine condamnent l'intervention américaine, violation du droit international et des objectifs de la Charte de l'ONU.

22.12.89 Les manifestations qui ont commencé à Timisoara le 16.12 et ont touché rapidement d'autres villes de Transylvanie puis Bucarest le 21, renversent avec l'aide de l'armée, le régime de M. N. Ceaucescu en Roumanie. Un conseil du front de salut national est formé sous la présidence de $\mathrm{M}$. Yon Iliescu, ancien secrétaire du C.C. du P.C. roumain, limogé en 1971.

Le 25.12, Nicolae et Elena Ceaucescu, arrêtés le 22 sont exécutés. Le 26.12, le porte parole du ministère des Affaires étrangères de la R.P. de Chine déclare que le gouvernement de Pékin respecte le choix du peuple roumain et maintiendra des relations d'amitié avec le nouveau Gouvernement.

23.12.89 Le ministre de l'éducation de la R.P. de Chine déclare au comité permanent de l'Assemblée nationale que les influences contre-révolutionnaires se font sentir dans l'enseignement et qu'à tous les niveaux, l'éducation devra être combinée avec du travail manuel.

27.12.89 L'Assemblée nationale du Vietnam adopte une nouvelle loi sur la presse qui interdit toute propagande contre le P.C. et contre le socialisme.

27.12.89 Un accord est signé à Hanoi entre le Vietnam et l'Italie, à propos de la coopération économique, technique et scientifique. L'Italie est ainsi le premier pays de la CEE à accorder une aide au Vietnam depuis le retrait des troupes vietnamiennes du Cambodge. 
29.12.89 Le C.C. du P.C. bulgare met fin à la politique d'assimilation forcée de la minorité turque.

Les Bulgares de souche protestent contre les droits reconnus aux minorités musulmanes.

29.12.89 La Pologne supprime les qualificatifs "socialiste" et "populaire" de son nom. Elle devient la République de Pologne et ne reconnaît plus un rôle dirigeant au P.C. (le parti ouvrier unifié polonais).

30.12.89 Le ministère des Affaires étrangères de la R.P. de Chine considère comme une violation grossière des accords de 1984 sur Hong Kong, l'attribution par le gouvernement de Londres de passeports assortis du droit de résidence en Grande Bretagne à 50.000 chefs de famille de Hong Kong et à 175.000 personnes qui dépendent d'eux.

1990

10.01.90 M. Li Peng, Premier ministre de la R.P. de Chine annonce la levée de la loi martiale à Pékin, instaurée le 20.05.89.

16.01.90 La demande d'admission au GATT faite par Taiwan le 01.01.90 est considérée par la R.P. de Chine comme illégale et destinée à crécr deux Chines.

16.01.90 Le Président de la Commission d'Etat du Plan de la R.P. de Chine en visite au Japon.

Les échanges à haut niveau ont ainsi repris entre la R.P. de Chine et la Japon et un nouvel emprunt est octroyé par le Japon à la R.P. de Chine, ce que la France fera aussi en février 1990.

19.01.90 Le gouvernement fédéral de l'Inde prend le contrôle de l'Etat du Cachemire. L'intensification de la répression contre les indépendantistes relance la tension entre l'Inde et le Pakistan. 
22.01.90 Les manifestations se multiplient en Allemagne de l'Est, elles exigent une rupture totale avec le communisme et réclament l'unification allemande.

Un nouveau gouvernement est formé le 5.2.90 dans lequel l'ancien parti socialiste unifié devenu le parti socialiste démocratique ne maîtrise que 16 ministères sur 35 .

Le 30.1. M. Gorbatchev reçoit le nouveau Premier ministre est allemand et accepte l'idée de l'unification des deux Etats allemands.

22.01.90 Le parti au pouvoir en Corée du Sud et deux formations centristes de l'opposition décident de se regrouper en un seul parti politique.

01.02.90 Le Front du salut national de Roumanie accepte de partager le pouvoir avec l'opposition. Les manifestants prennent d'assaut et saccagent l'immeuble du gouvernement à Bucarest.

07.02.90 Le C.C. du P.C. de l'URSS approuve le projet de programme présenté par $M$. Gorbatchev, y compris l'abandon du rôle dirigeant du P.C. et le passage à un régime présidenticl démocratique.

08.02.90 Des précisions sont publiées dans la presse de la R.P. de Chine à propos du système de coopération entre les partis politiques: tous doivent accepter le rôle dirigeant du P.C. et coopérer avec lui. Ceux qui s'opposent aux quatre principes cardinaux du régime et ceux qui mettent en danger le pouvoir de l'Etat, sont interdits.

13.02.90 Les quatre puissances garantes du statut de l'Allemagne (EtatsUnis, France, Grande Bretagne et l'URSS) et les deux Etats allemands décident d'ouvrir des discussions sur les problèmes extérieurs de l'unification de l'Allemagne.

13.02.90 les Ministres des Affaires Etrangères de l'OTAN et du Pacte de Varsovie réunis à Ottawa, concluent deux accords :

- sur l'unification de l'Allemagne 
- sur le plafond de 195.000 hommes pour les troupes américaines et soviétiques dans la zone centrale de l'Europe.

18.02.90 Le parti libéral démocrate conserve la majorité absolue à la Chambre basse du Japon (275 des 512 sièges) en dépit d'une nette progression du parti socialiste (136 sièges).

18.02.90 Publication du projet de loi fondamentale de la future région administrative spéciale de Hong Kong.

24.02.90 Les indépendantistes remportent dès le premier tour la majorité des sièges au Parlement de la République de Lituanie.

25.02.90 Mme Violetta Chamorro remporte $54.7 \%$ des suffrages sur $M$. Daniel Ortega ( $40.8 \%$ ) a l'élection présidentielle du Nicaragua. Les guerrilleros de la contra acceptent leur démobilisation.

28.02.90 Des relations commerciales directes sont établies entre Taiwan et l'URSS ainsi qu'entre Taiwan et l'Albanie. La R.P. de Chine considère que son gouvernement doit être consulté avant que soient établis des vols directs entre Taiwan et l'URSS.

04.03.90 Les réformateurs et les radicaux remportent d'importants succès aux élections locales en Russie, en Ukraine et en Biélorussie.

06.03.90 Le chancelier Kohl accepte de reconnaître l'intangibilité de la frontière germano-polonaise sur l'Oder-Neisse.

08.03.90 Le ministère des Affaires étrangères de la R.P. de Chine déclare que la situation au Cambodge constitue le principal obstacle à la normalisation des relations avec le Vietnam. Il ne s'agit pas de rechercher l'appui de Hanoï pour condamner la "perestroïka" soviétique.

11.03.90 Le parlement lituanien adopte une déclaration d'indépendance. Moscou applique des pressions militaires et des sanctions économiques à partir du 18.03. 
13.03.90 Le congrès des députés de l'URSS approuve des amendements a la Constitution qui instaurent un régime présidentiel, la pluralité des partis et la propriété privée.

14.03, M. Gorbatchev est élu président pour 5 ans.

14.03.90 Le Secrétaire général du P.C. chinois en visite en Corée du Nord est reçu par le Président Kim Il sung.

18.03.90 Les nationalistes remportent les élections locales en Estonie et en Lettonie. Les parlements de ces républiques votent le 30.03 et le 04.05 en faveur de l'indépendance moyennant une longue transition.

18.03.90 Le résultat des premières élections législatives libres depuis 1933 en RDA sont interprétés comme un plébiscite en faveur de l'unification rapide de l'Allemagne.

M. Lothar de Maizière (chrétien-démocrate) forme le 12.4 un gouvernement de coalition avec les sociaux démocrates et les libéraux.

20.03.90 Le Ministre des Affaires Etrangères de Chine, à la NouvelleDelhi déclare que son pays recherche des liens étroits avec l'Inde.

20.03.90 La 3ème session de la 7ème législature de la R.P. de Chine s'ouvre a Pékin.

Le Premier ministre Li Peng réitère l'engagement à long terme dans un programme de réformes et d'ouverture mais annonce des mesures immédiates de plus grande centralisation et de planification et de plus étroite intégration des réformes aux quatre principes cardinaux.

Le 21.3.90, l'Assemblée nationale accepte la démission de $\mathrm{M}$.

Deng Xiaoping de la présidence de la Commission militaire centrale de l'Etat. Le 3.4.90, M. Jiang Zemin est nommé président. Malgré le déficit du budget de l'Etat, les allocations pour l'agriculture, l'enseignement et la défense nationale sont augmentées.

4.4.90 l'Assemblée adopte la loi fondamentale de la région spéciale de Hong Kong. 
21.03.90 L'indépendance de la Namibie est officiellement proclamée.

22.03.90 La R.P. de Chine et la Namibie établissent des relations diplomatiques.

23.03.90 Le parlement de Mongolie vote l'abolition du rôle dirigeant du parti populaire révolutionnaire mongol. Après la démission des dirigeants du parti (12.3) et de l'Etat (15.3), des réformateurs les remplacent.

24.03.90 Le parti travailliste de M. Bob Hawke au pouvoir depuis 1983 remporte les élections législatives en Australie pour la 4ème fois consécutive.

25.03.90 La majorité se prononce en faveur de la démocratie et du rejet de l'ancien régime lors des premières élections législatives libres en Hongrie.

06.04.90 Signature d'un accord intérimaire entre les Etats-Unis et le Japon à propos des entraves structurelles aux échanges commerciaux.

Le 27.04.90, les Etats-Unis rayent le Japon de la liste noire commerciale.

07.04.90 L'ambassadeur de la R.P. de Chine au Lesotho déclare que son pays devra rompre ses relations diplomatiques avec ce pays en raison de son rétablissement de liens diplomatiques avec Taiwan.

07.04.90 Réduction de la bureaucratie en Mongolie par fusion de ministères et commissions nationales.

08.04.90 Le roi Birendra du Népal accepte le rétablissement du multipartisme après des agitations sévèrement réprimées.

08.04.90 Le Président de l'Irak menace de mettre à feu la moitié d'Israël si ce pays tente quoique ce soit contre l'Irak.

08.04.90 L'opposition non communiste obtient $55 \%$ des suffrages aux élections législatives libres de Slovénie. 
Les 22 avril, 6 et 7 mai, les élections sont remportées en Croatie par l'Union démocratique croate ( $2 / 3$ des sièges).

09.04.90 Le ministre japonais des Finances étudie la reprise des prêts, gelés depuis 1989, à la R.P. de Chine.

10-13.04.90 Le Congrès extraordinaire du parti révolutionnaire du peuple mongol a élu un nouveau comité central, un nouveau président, M. G. Orchirbat et a défini sa "ligne de renouveau" : construction d'un socialisme humain et démocratique, pluralisme politique.

11.04.90 L'ambassadeur de la R.P. de Chine en France proteste contre le droit d'asile politique qu'accorde la France a des participants aux événements de mai-juin 1989. "Criminels recherchés par la sûreté chinoise pour la part prise dans la rébellion contrerévolutionnaire".

11.04.90 Le Président G. Bush promulgue un décret qui autorise les ressortissants chinois à rester aux Etats-Unis.

13.04.90 Les Présidents de la Guinée Equatoriale et de la République Centre africaine sont en visite en R.P. de Chine.

14.04.90 Un accord de coopération économique et technique est conclu a Pékin entre la Guyane et la R.P. de Chine à l'issue d'une visite du Ministre des Affaires Etrangères de Guyane en R.P. de Chine.

15-16.04.90 La R.P. de Chine fait face à une rébellion armée "contre révolutionnaire" près de Kashgar au Xinjiang (le Turkestan chinois).

Le 21.4, nouvelle annonce par la presse chinoise d'une rébellion contre révolutionnaire au Xinjiang qui aurait eu lieu le 5.4. et n'aurait pas de raison religieuse ni nationale.

20-27.04.90 A la réunion du groupe de liaison sino-britannique à propos de Hong Kong, le représentant de la R.P. de Chine déclare que le projet de déclaration des droits proposé par Londres pour sa colonie soulève de nombreux problèmes et notamment celui 
de la convergence de ce texte avec la loi fondamentale de la région administrative spéciale à laquelle la déclaration ne peut être supérieure.

13.06.90 Le Parlement de Londres adopte une loi sur la nationalité britannique de personnes établies à Hong Kong, loi que la R.P. de Chine considère en contradiction avec les accords de 1984.

21.04.90 Le Premier ministre de R.P. de Chine en URSS. MM. Rijkov et Li Peng concluent un programme à long terme de coopération économique, scientifique et technique ainsi qu'un accord de coopération en matière d'utilisation pacifique de l'énergie nucléaire.

les Ministres des Affaires Etrangères des deux pays définissent les principes qui guident les réductions mutuelles des forces armées et les mesures destinées à rétablir la confiance dans la zone frontalière entre l'URSS et la R.P. de Chine.

La R.P. de Chine accorde un crédit à l'URSS pour lui faciliter l'achat de biens de consommation.

L'URSS octroie des crédits à la R.P. de Chine pour la construction de deux centrales nucléaires.

$M$. Gorbatchev en recevant le ministre Li Ping déclare qu'il n'existe pas de formule universelle pour l'application des idées du socialisme et que les différences de vues qui continuent à exister entre les deux pays n'empêchent pas le développement de leurs relations bilatérales.

23.04.90 Le Chancelier Kohl accepte la parité pour l'échange des marks d'Allemagne de l'Est contre les deutsmarks pour les salaires, les retraites et une partie de l'épargne. L'union monétaire des deux Allemagne est fixée au 1.7.90.

28.04.90 Début des conversations entre représentants au pouvoir et de l'opposition en Mongolie, en vue de former un Conseil populaire chargé de contrôler les prochaines élections et de mettre en place une deuxième chambre servant de législature suprême.

01.05.90 Des milliers de manifestants conspuent $M$. Gorbatchev à l'issue de la célébration du 1er mai sur la place Rouge à Moscou. 
01.05.90 La loi martiale est levée au Tibet, elle y avait été imposée le 8.3.1989.

02.05.90 Visite en R.P. de Chine du Ministre des Affaires Etrangères de l'Equateur; signature de quatre accords de coopération économique et culturelle.

03.05.90 Le montant de la dette extérieure de la R.P. de Chine à la fin de 1989 s'élève à 41.3 milliards de dollars.

03.05.90 La R.P. de Chine accuse Taiwan de persévérer dans sa politique de "deux Chines" ou "d'une Chine et un Taiwan".

27.05.90 Chaque pays ne peut avoir qu'un seul gouvernement souverain; le concept d'"un pays, deux gouvernements" défendu par Taiwan est donc inacceptable déclare $M$. Jiang Zemin. Celui-ci propose des discussions sur un plan d'égalité entre P.C.C. et K.M.T. le 11.06.90.

04.05.90 Première visite en R.P. de Chine d'un Président du comité permanent de l'Assemblée nationale de la République populaire de Mongolie.

Le Secrétaire général du P.C.C. exprime sa joie à propos de la normalisation des relations entre le P.C.C. et le parti révolutionnaire du peuple mongol. Des accords de coopération scientifique, technique et de protection de l'environnement sont signés.

05.05.90 La première réunion ministérielle de la Conférence dite " $2+4$ " (RDA-RFA + USA, France, Grande Bretagne et URSS) sur les aspects extérieurs de l'unification allemande se tient à Bonn.

06.05.90 La R.P. de Chine considère que la tournée de neuf jours faite par le Premier ministre japonais M. T. Kaifu en Inde, au Bangladesh, au Pakistan, à Sri Lanka et en Indonésie est une preuve de l'évolution de la diplomatie japonaise en Asie Pacifique; il s'agit d'établir une zone d'influence grâce à une aide accrue mais aussi d'affirmer le rôle que le Japon joue dans les problèmes régionaux et globaux. 
06.05.90 M. Wan Li, président du comité permanent de l'Assemblée nationale populaire de R.P. de Chine en visite au Pakistan.

07.05.90 Le Président des Emirats Arabes Unis accompagné de son Ministre des finances fait une visite en R.P. de Chine.

08.05.90 Le Parlement albanais vote plusieurs mesures de libéralisation, M. Ramiz Alia, dirigeant du P.C. affirme le 12.05 que la démocratisation est "irréversible".

09.05.90 Le Vietnam annonce que des conversations non officielles ont eu lieu à Pékin pour discuter du Cambodge et normaliser les relations avec la R.P. de Chine.

Le 10.5, la R.P. de Chine fait savoir que la situation au Cambodge continue à constituer un obstacle principal et que l'aide aux forces de la triple coalition de la résistance sera maintenue tant que le Vietnam n'aura pas achevé le retrait de ses troupes.

10.05.90 Plus de 200 personnes arrêtées pour leur participation aux troubles de mai et juin 1989 sont libérées.

Les autorités de la R.P. de Chine déclarent le 6.6.90 que 860 citoyens ont été libérés et qu'il resterait 355 incarcérés.

11.05.90 Le Premier ministre de la Barbade, en visite officielle en R.P. de Chine y conclut un accord de cooperation économique et technique.

11-14.05.90 Visite en R.P. de Chine du Président Mubarak d'Egypte. Des accords sont signés à propos de l'augmentation des échanges commerciaux et de la création de vols directs entre Pékin et Le Caire.

13.05.90 Le Président de la R.P. de Chine, M. Yang Shang Kun se rend en visite d'Etat au Mexique, au Brésil, en Uruguay, en Argentine et au Chili. Le 30.5.90, il termine sa tournée par une visite d'Etat aux Iles Fidji.

14-19.05.90 Pourparlers à Manille sur l'avenir des bases américaines aux Philippines dont le bail expire en septembre 1991. 
Les 18-21.09.90, les Etats-Unis acceptent le principe d'une transition avec réduction de leurs moyens et certaines activités économiques dans les bases.

15.05.90 Deuxième étape du retrait des troupes soviétiques de Mongolie: 26.000 soldats quitteront le pays avant le 01.08 .90 et le retrait sera achevé en 1992.

15.05.90 Le Président de la R.P. de Chine, M. Yang Shang Kun en visite au Mexique définit les principes qui régissent les relations de son pays avec l'Amérique latine : liens d'amitié et de coopération avec tous les pays de la région, même en l'absence de relations diplomatiques, sur base des cinq principes de la coexistence pacifique, développement des échanges commerciaux et de la coopération économique sur base de l'égalité des parties et du bénéfice mutuel, respect réciproque des traditions et des valeurs des parties.

15.05-03.06.90 Visite officielle du Premier Ministre de Singapour en Turquie, en France, en Grande Bretagne et en Allemagne.

20.05.90 Visite en R.P. de Chine de l'ancien chancelier de RFA, M. H. Schmidt, première personnalité politique à se rendre en Chine depuis le 4.6.89.

20.05.90 M. Ion Iliescu remporte l'élection présidentielle en Roumanie avec $85,07 \%$ des suffrages et le F.S..N. qu'il dirige, 233 des 348 sièges des députés et 92 des 119 sièges de sénateurs.

20.05.90 Le Président de Taiwan appelle le gouvernement de Pékin au dialogue en vue d'une réunification. Il rompt ainsi avec 40 années de non reconnaissance du régime de la R.P. de Chine. Le 20.05.90, le Ministre taiwanais de la défense, le Général Hau Peir-tsun est nommé Premier Ministre en remplacement de $\mathrm{M}$. Lee Huan.

22.05.90 Le Yemen du Sud, marxiste, et celui du Nord, conservateur, sont unifiés avec pour Président, le général Ali Abdallah Saleh, chef de l'Etat du Nord Yemen depuis 1978. 
23.05.90 Investiture par le Parlement Hongrois du gouvernement de coalition de centre droit formé par M. Joseph Antall, Président du Forum Démocratique.

23.05.90 M. Kim Il-sung est réélu chef de l'Etat en Corée du Nord, pour 4 ans.

24.05.90 L'Empereur Akihito exprime ses plus profonds regrets pour les souffrances endurées par les Coréens à la suite des actions passées du Japon, lors du banquet offert en l'honneur du Président la Corée du Sud, M. Roh Tae Woo.

24.05.90 Le Président des Etats-Unis annonce l'extension pour un an du statut de nation la plus favorisée à la R.P. de Chine.

25.5.90. Le Premier ministre Li Peng se réjouit de cette décision, favorable aux intérêts de la R.P. de Chine, des Etats-Unis et à la prospérité et à la stabilité de Hong Kong.

26.05.90 Un journal de Hong Kong rapporte que la R.P. de Chine a fait exploser une bombe à hydrogène de la deuxième génération. Ce test, réalisé sous terre, est considéré comme un rappel du rôle de la R.P. de Chine dans les relations internationales, à la veille du sommet URSS-Etats-Unis.

27.05.90 L'opposition remporte les premières élections multipartites organisées en Birmanie (Myanmar) par les militaires au pouvoir depuis 1962. La ligue nationale pour la démocratie de Mme Aung San Suu Kyi obtient 396 des 485 sièges. La junte militaire refuse cependant d'abandonner le pouvoir.

29.05.90 Les statuts de la Banque européenne pour la reconstruction et le développement sont adoptés par les $\mathbf{4 2}$ membres fondateurs.

29.05.90 Constitution d'un groupe de travail pour remédier à l'excédent commercial du Japon vis-à-vis de la CEE, à Bruxelles.

28-30.05.90 Les Chefs d'Etat de l'Algérie, du Maroc, de la Syrie, du Liban et d'Oman refusent d'assister à Bagdad au sommet extraordinaire de la Ligue Arabe convoqué à la demande de 
l'OLP pour examiner "les dangers de l'immigration des juifs soviétiques en Israël".

31.05.90 Le groupe sud-coréen Samsung annonce la conclusion d'un important accord avec l'URSS pour la modernisation des télécommunications soviétiques.

31/05-02.06.90 Deuxième rencontre au sommet MM. Bush-Gorbatchev aux Etats-Unis. Un accord intervient en matière d'interdiction de la production d'armes chimiques, les relations commerciales entre l'URSS et les Etats-Unis sont normalisées et un projet de traité sur les armes nucléaires est arrêté.

01.06.90 Première visite d'une délégation militaire soviétique en R.P. de Chine depuis 1960.

02.06.90 Visite en Egypte et en Jordanie du Ministre de la défense nationale de la R.P. de Chine.

02.06.90 La R.P. de Chine rompt ses relations diplomatiques avec la Guinée Bissau qui a rétabli des liens diplomatiques avec Taiwan.

04.06.90 A l'issue du sommet américain-soviétique, M. Gorbatchev rencontre à San Francisco M. Roh Tae-Woo, Président de la République de Corée (S).

07.06.90 Les dirigeants des sept pays membres du Pacte de Varsovie décident de reconsidérer le caractère et les fonctions de leur alliance afin de lui donner un rôle plus politique que militaire. La Hongrie annonce sa volonté de quitter le Pacte avant la fin de 1991.

8-9.06.90 96\% des électeurs inscrits participent aux premières élections législatives libres depuis 1946 en Tchécoslovaquie.

Le Forum civique du Président V. Havel et son homologue slovaque remportent la majorité absolue au Parlement fédéral. 
10-17.06.90 Le parti socialiste (ex communiste) obtient la majorité absolue avec 211 sièges sur 400 aux élections législatives libres en Bulgarie.

12.06.90 Les députés de la fédération de Russie sous la présidence de $M$. Boris Eltsine proclament par 907 voix contre 13 la "souveraineté d'Etat" de leur République.

12.06.90 Le Gouvernement australien accorde un droit temporaire de résidence pour une période non spécifiée allant de 6 mois à 10 ans, aux quelques 20.000 étudiants chinois qui étaient sur son territoire avant le 4.6.1989.

13.06.90 Des émeutes éclatent à Bucarest après l'évacuation par la police de la place de l'Université occupée depuis le 22.4 par des manifestants.

Les 14 et 15 juin, des mineurs de houille appelés à Bucarest pour rétablir l'ordre font régner la terreur et saccagent les locaux de partis politiques, de journaux et d'universités.

21.06.90 Après le limogeage de hauts fonctionnaires et d'un ministre, le gouvernement du Cambodge affirme avoir déjoué un "coup d'Etat réactionnaire".

24.06.90 Le gouvernement israélien annonce que les immigrants soviétiques (près de 50.000 en moins de 6 mois) sont installés a l'intérieur des frontières de 1967.

25.06.90 M. Fang Lizhi et son épouse sont autorisés à quitter la R.P. de Chine pour suivre un traitement médical à l'étranger, après avoir admis leur opposition aux quatre principes cardinaux. Ils se rendent en Grande Bretagne.

26.06.90 Le Président du Tchad, M. H. Habré arrive en visite en R.P. de Chine.

29.06.90 Le Parlement lituanien accepte de suspendre la déclaration d'indépendance du 11 mars et l'URSS lève les sanctions imposées à la Lituanie depuis le 18 avril. 
29.06.90 Le Président H. Ershad du Bangladesh en visite officielle en R.P. de Chine.

01.07.90 L'union monétaire et sociale entre RFA et RDA entre en vigueur.

08.07.90 Le quartier des ambassades à Tirana est cerné par la police et l'armée alors que des milliers d'albanais sont réfugiés dans les ambassades européennes.

Les autorités accordent des passeports à 4.786 personnes. A partir du 12.7, commence l'évacuation par bateaux vers Brindisi.

10.07.90 M. Gorbatchev est rélu Secrétaire général du P.C. de l'URSS lors de son 28ème congrès.

11.07.90 Les sept pays industrialisés expriment, lors de leur sommet à Houston, leur volonté d'assouplir les sanctions prises contre la R.P. de Chine.

14.07.90 Le Secrétaire général de l'OTAN s'entretient à Moscou avec M. Gorbatchev.

15-16.07.90 $\mathrm{M}$. Kohl en visite en URSS conclut un accord avec $\mathrm{M}$. Gorbatchev. Moscou lève son opposition à l'appartenance de la future Allemagne unie à l'OTAN.

Bonn accepte de limiter les effectifs de la future armée allemande a 370.000 hommes et de financer le retrait des 380.000 soldats soviétiques de RDA avant la fin 1994.

Une substantielle aide est fournie par Bonn à l'URSS; cet accord est entériné le 17.7 par la Conférene $(2+4)$ de Paris.

La Pologne obtient des Allemands l'engagement que l'intangibilité de la frontière germano-polonaise le long de la ligne Oder Neisse sera garantie par un traité.

Ce traité est paraphé le 14.11.90 a Varsovie.

16.07.90 Le parlement de l'Ukraine adopte une déclaration de souveraineté.

Celui de Biélorussie fait de même le 27.7 et celui d'Arménie, le 23.8. 
17.07.90 Le Japon rétablit son aide a la R.P. de Chine.

19.07.90 Le Président de la Commission des communautés européennes est reçu à Moscou.

21.07.90 L'Arabie Saoudite établit des relations diplomatiques avec la R.P. de Chine.

23-25.07.90 Visite en URSS du Premier ministre de l'Inde.

27.07.90 Sous la pression de l'Irak, l'OPEP fixe à 21 dollars le prix de référence du baril de pétrole brut, gelé à 18 dollars depuis décembre 1986.

29.07.90 Les premières élections législatives multipartites sont organisées en République de Mongolie.

Le parti au pouvoir depuis 1924 remporte 357 des 430 sièges à pourvoir.

30.07.90 Après une rupture depuis 1961, les relations diplomatiques entre l'URSS et l'Albanie sont rétablies.

01.08.90 Nouvelle lutte contre la délinquance à caractère politique, au Vietnam. Elle concerne principalement les organisations et les individus qui font de la propagande pour le pluralisme politique, la démocratie extrémiste et l'opposition au régime.

01.08.90 Le dirigeant de l'opposition bulgare, M. Jelevic Jeliv est élu président de la République.

Le 27.8 les manifestants incendient le siège du parti socialiste à Sofia.

02.08.90 L'armée irakienne envahit le Koweit.

Le Conseil de Sécurité de l'ONU exige le retrait immédiat et inconditionnel des forces irakiennes et des mesures économiques et financières de rétorsion sont décidées contre l'Irak par les pays Occidentaux. 
06.08.90 Le Conseil de Sécurité de l'ONU adopte par 13 voix sur 15 (Cuba et Yemen s'abstiennent) une résolution qui instaure un embargo commercial, financier et militaire contre l'Irak.

07.08.90 Les Etats-Unis lancent l'opération "Bouclier du désert".

Des troupes, des avions et des blindés sont envoyés en Arabie Saoudite par les Etats-Unis; la Grande Bretagne et la France annoncent l'envoi de forces militaires.

08.08.90 Les relations diplomatiques suspendues depuis 1967, sont rétablies entre l'Indonésie et la R.P. de Chine.

08.08.90 Bagdad annonce la fusion totale et irréversible de l'Irak et du Koweit.

Le 9.8., le Conseil de Sécurité de l'ONU adopte à l'unanimité une résolution qui déclare nulle et non avenue cette annexion.

10.08.90 Un sommet arabe extraordinaire réuni au Caire décide l'envoi d'une force arabe pour défendre l'Arabie Saoudite.

11.08.90 L'Egypte, la Syrie, le Maroc, le Pakistan, le Bangladesh envoient des troupes soutenir l'Arabie Saoudite.

12.08.90 Le Président irakien préconise une solution globale de tous les problemes d'occupation dans la région.

15.08.90 L'Irak accepte les conditions mises par l'Iran à un accord de paix. Son armée se retire de tout le territoire iranien occupé depuis août 1988 et l'échange des prisonniers de guerre commence entre l'Iran et l'Irak.

18.08.90 L'Irak fait savoir que les "ressortissants des nations agresseurs" seront retenus en Irak et au Koweit. Occidentaux et Japonais seront utilisés comme boucliers humains dans des installations militaires et des centres stratégiques.

Le 18.8, le Conseil de Sécurité de l'ONU exige, par un vote unanime, que l'Irak autorise et facilite le départ des étrangers du Koweit et de l'Irak. 
28.08.90 L'Irak décrète que le Koweit devient la 19ème province irakienne.

Les femmes et les enfants étrangers retenus en otages sont autorisés à partir.

28.08.90 Les cinq membres permanents du Conseil de Sécurité de l'ONU adoptent un plan de paix pour le Cambodge qui définit le cadre d'un règlement pacifique du conflit. Un conseil national suprême est formé le 11.9 avec la participation des quatre factions cambodgiennes mais des désaccords à propos de la présidence de ce conseil continuent à bloquer le processus de paix.

31.08.90 Le traité d'unification des deux Allemagne est paraphé à Berlin Est et est ratifié à Paris le 20.9.90.

2-7.09.90 Tournée en Extrême Orient du Ministre soviétique des Affaires étrangères : en R.P. de Chine, en Corée du Nord et au Japon.

3-7.09.90 Visite secrète en R.P. de Chine du Secrétaire général du P.C. vietnamien accompagné d'un ancien Premier ministre. 19.09 Le Général Vo Nguyen Giap est reçu à Pékin.

04.09.90 Etablissement de relations diplomatiques entre l'URSS et Kiribati.

4-7.09.90 Les Premiers ministres de la Corée du Nord et de la Corée du Sud se rencontrent à Séoul et entament un véritable dialogue. La Corée du Sud demande un accord sur des mesures de réconciliation alors que la Corée du Nord veut une déclaration conjointe de non agression et un engagement à désarmer.

06.09.90 Visite en R.P. de Chine du Vice Premier ministre irakien; la R.P. de Chine veut le rétablissement de l'intégrité territoriale, de l'indépendance et de la souveraineté du Koweit, par une solution arabe sans recours à la force.

08.09.90 Signature à Moscou par les Ministres des Affaires Etrangères de l'URSS et de la R.F.A. d'un traité de bon voisinage, de partenariat et de coopération entre les deux pays. 
08-09.09.90 MM. Bush et Gorbatchev se rencontrent à Helsinki. Ils se déclarent unis et déterminés à montrer que l'agression ne peut pas être payante tout en exprimant leur préférence pour une solution pacifique.

9-10.09.90 Le ministre irakien des Affaires étrangères se rend en Iran et obtient la normalisation des relations diplomatiques rompues depuis 1980 mais l'Iran n'accepte pas d'aider l'Irak à contourner l'embargo.

11.09.90 Début de la campagne d'épuration du P.C. vietnamien.

12.09.90 Signature à Moscou du traité qui règle les aspects extérieurs de l'unification allemande.

13.09.90 Le gouvernement japonais décide de fournir deux milliards de dollars pour le déploiement des forces unilatérales dans le Golfe et un prêt de deux milliards de dollars aux pays touchés par la crise.

17.09.90 Des relations diplomatiques sont rétablies entre l'Arabie Saoudite et l'URSS, rompues depuis 1939.

27.11.90 un prêt saoudien de 4 milliards de dollars est accordé à l'URSS.

22.09-07.10.90 Les 11ème Jeux asiatiques se déroulent a Pékin. 37 pays y participent, parmi lesquels Taiwan (Taibei-Chine), le Vietnam et le Koweit.

L'Irak en a été exclu le 20.9 pour son invasion du Koweit.

24.09.90 Le parlement soviétique autorise le Président Gorbatchev à légiférer par décrets pendant 18 mois pour introduire en URSS l'économie de marché.

25.09.90 Le Conseil de Sécurité adopte par 14 vois sur 15 (Cuba seul s'y oppose) une résolution qui étend l'embargo contre l'Irak au trafic aérien. 
27.09.90 Rencontre à New York des ministres des Affaires étrangères des Etats-Unis et du Vietnam, entretiens sur la normalisation des relations.

27.09.90 Les relations diplomatiques rompues en mars 1989 sont rétablies entre la Grande Bretagne et l'Iran.

28.09-01.10.90 Visite à New York du Premier ministre japonais. Entretiens avec le Président G. Bush sur la contribution japonaise à la force multinationale dans le Golfe.

28.09.90 L'Agence américaine pour le développement international accorde au Cambodge une aide humanitaire de 2 millions de dollars.

30.09.90 La Corée du Sud et l'Union Soviétique établissent des relations diplomatiques.

Du 13 au 16 décembre 1990, le Président de la Corée du Sud, M. Roh Tae-woo se rend en visite à Moscou.

01.10.90 La Banque d'Etat du Vietnam annonce un nouveau taux de change pour le dong : $1 \$$ US $=5.800$ dong.

01.10.90 Le Président G. Bush déclare à l'Assemblée générale de l'ONU que l'évacuation inconditionnelle du Koweit par l'Irak créerait des occasions qui permettraient de résoudre le conflit qui oppose les Arabes à Israël.

01.10.90 Adoption en URSS d'une loi qui instaure la liberté de conscience et de religion. L'Etat n'a plus le droit de propager l'athéisme.

01.10.90 Les Etats Unis suspendent toute aide économique et militaire au Pakistan, parce qu'ils craignent la mise au point d'armes nucléaires par les autorités d'Islamabad.

03.10.90 Le ministre laotien des Affaires étrangères rencontre à l'ONU le secrétaire d'Etat James Baker.

C'est le premier contact a haut niveau entre le Laos et les Etats Unis depuis 1975. 
2-6.10.90 M. T. Kaifu, le Premier ministre du Japon, entreprend une tournée dans le Golfe : Egypte, Jordanie, Irak, Turquie, Arabie Saoudite, Oman auxquels il offre une aide financière.

Le 12.10, il demande au Parlement japonais l'envoi d'un corps de paix pour participer aux missions de l'ONU (transfert, communication, réparation...).

03.10.90 Les deux parties de l'Allemagne ne forment plus que la RFA. Le 1.10.90, les quatre puissances qui ont gagné la guerre en 1945 signent une déclaration qui confère la pleine souveraineté à la nouvelle Allemagne unifiée.

03.10.90 MM. Baker et Chevardnadze annoncent à New York qu'un accord a été atteint à propos d'un traité sur le déploiement des armes conventionnelles en Europe, qui devra être approuvé par l'OTAN et le Pacte de Varsovie.

Des plafonds sont prévus pour chacune des deux alliances et pour chaque pays membre ( $30 \%$ de l'ensemble au maximum).

03.10.90 Des relations diplomatiques sont établies entre la R.P. de Chine et Singapour. Il n'y a plus dans l'ASEAN que Brunéi qui n'ait pas reconnu la R.P. de Chine. Du 16 au 24.10, M. Lee Kuan Yew est pour la 5 ème fois l'hôte officiel de la R.P. de Chine.

04.10.90 Une tentative de coup militaire a lieu sur deux bases militaires dans le nord de Mindanao aux Philippines.

Le colonel Noble et ses compagnons se rendent le 6.10.90.

05.10.90 Le "petit hural" c'est-à-dire le comité permanent de la nouvelle assemblée nationale élue en Mongolie forme, au cours de sa premiere session, un gouvernement qui comprend des représentants de quatre partis politiques et compose une commission chargée de rédiger une nouvelle Constitution.

7-11.10.90 Visite en Inde du Premier ministre du Cambodge.

07.10.90 Création à Taiwan du comité de réunification nationale présidé par M. Lee Teng-hui. Ce sera la plus haute instance consultative en matière de politique à l'égard de la Chine continentale. 
09.10.90 Le Soviet suprême ratifie les traités déjà ratifiés par le Sénat des Etats-Unis le 25.9.90 sur les interdictions des essais d'armements stratégiques de 1974 et sur la limitation des explosions nucléaires souterraines de 1976.

10-13.10.90 Au Vietnam, 38 membres de mouvements de résistance sont condamnés à des peines de prison, de 3 ans à la perpétuité, pour "activités de trahison".

10-15.10.90 De violentes manifestations anti-gouvernementales à Dacca, capitale du Bangladesh, exigent la démission du Président $\mathrm{H}$. Ershad et de nouvelles élections.

11.10.90 Deux marins japonais détenus depuis 1983 en Corée du Nord sont libérés alors qu'ils avaient été condamnés en 1987 à 15 ans d'emprisonnement.

C'est un geste de conciliation de Pyongyang après la visite en Corée du Nord, en septembre 1990, de représentants du parti libéral démcorate et du parti socialiste du Japon.

13.10.90 Un traité de bon voisinage et de coopération est conclu entre la RFA et l'URSS.

13-25.10.90 Violents combats au Cambodge opposant troupes gouvernementales et forces armées de la résistance, au nord ouest et au sud du pays.

15.10.90 Le Prix Nobel de la paix 1990 est conféré au Président M. Gorbatchev pour ses contributions multiples et décisives aux relations Est-Ouest et pour la plus grande ouverture qu'il a introduite dans la société soviétique.

15.10.90 Une résolution de l'Assemblée générale des Nations Unies appelle les dirigeants du Cambodge à coopérer et invite le Conseil national suprême à envoyer son représentant pour occuper le siège du Cambodge à l'ONU. Le désaccord entre la résistance et le gouvernement s'accompagne de combats sur le terrain. 
16-19.10.90 Une délégation du gouvernement de Corée du Sud conduite par le Premier ministre se rend en visite en Corée du Nord, en réponse aux conversations du 4 au 7 septembre tenues à Séoul. Elle est reçue par le Président Kim Il-sung le 18.10. Aucun accord n'est atteint à propos de la participation à l'ONU : la Corcé du Sud souhaite l'entrée des deux Corée à l'ONU tandis que la Corée du Nord souhaite que deux délégations partagent un seul siege a l'ONU.

20.10.90 Un accord commercial est signé entre la R.P. de Chine et la Corée du Sud.

Des bureaux économiques faisant office de liaison entre les deux gouvernements sont ouverts à Pékin et à Séoul sans qu'aient été nouées des relations diplomatiques.

20-21.10.90 Des élections générales à la Chambre basse du Parlement fédéral ont lieu en Malaysie.

M. Mahathir, Premier ministre depuis 1981, obtient un troisieme mandat, la coalition du front national gagne 127 des 180 sièges à pourvoir.

20.10.90 En Birmanie, tous les partis d'opposition doivent faire allégeance au décret du $27.7 .90 \mathrm{du}$ Conseil étatique de la loi et de la restauration de l'ordre, le SLORC, au pouvoir.

Ce décret confie l'exercice de tous les pouvoirs au SLORC et demande la rédaction d'une nouvelle Constitution qui sera soumise à référendum avant le tranfert du pouvoir à un gouvernement civil.

L'agitation continue et le 21.10 la répression s'exerce a l'encontre des moines bouddhistes.

21-27.10.90 Visite à Tokyo du Ministre vietnamien des Affaires étrangères. Première visite officielle depuis 1978.

22.10.90 Le Conseil des ministres des Affaires étrangères des membres de la Communauté économique européenne décide de lever les sanctions diplomatiques et économiques prises contre la R.P. de Chine et contre l'Iran.

L'embargo sur la livraison d'armes et l'interdiction de coopération militaire restent en vigueur à l'égard de la R.P. de 
Chine. Le Conseil décide d'établir des relations diplomatiques et économiques avec le Vietnam, rompues depuis l'invasion du Cambodge en décembre 1978.

23.10.90 Le Président de l'Union indienne refuse de dissoudre le Parlement après qu'un parti se soit retiré de la coalition, à la suite des tensions communautaires qui continuent à secouer le nord du pays. Une motion de défiance contre le gouvernement le 7.11.90 contraint M. Singh, Premier ministre, à démissionner.

Le 10.11.90 un gouvernement conduit par M. Chandra Shekhar est formé, avec l'appui au Parlement du Parti du congrès.

24.10.90 L'URSS procède à un essai nucléaire souterrain en Nouvelle Zemble.

24.10.90 Le Soviet Suprême de l'URSS adopte une législation d'urgence qui réitère la suprématie des lois de l'URSS sur celles des républiques fédératives.

24-27.10.90 Aux élections générales pour le renouvellement de l'Assemblée nationale et des assemblées locales au Pakistan, le parti du peuple pakistanais qui est au pouvoir est battu par l'alliance démocratique musulmane.

Le Premier ministre Mme $B$. Bhutto avait été accusée de fraudes et démise de ses fonctions le 6 août 90 .

Le 01.11.90, l'alliance nomme M. Mian Nawas Sharif, Premier ministre.

26.10.90 Visite de M. Gorbatchev en Espagne.

Accord d'aide économique et octroi de crédits à l'URSS.

27-28.10.90 Des élections législatives générales ont lieu en Nouvelle Zélande. Le parti national gagne $49 \%$ des voix et 68 des 97 sieges dans la Chambre unique du Parlement.

M. Jim Bolger devient Premier ministre, après 6 années de gouvernement travailliste. 
28-29.10.90 M. Gorbatchev en visite en France signe un traité d'amitié et de coopération, un programme de 5 ans pour la formation et la recherche scientifique. La France accorde des crédits à l'URSS. M. Gorbatchev à Paris réclame la convocation d'une conférence internationale pour résoudre la crise du Golfe et déclare que "l'option militaire est inacceptable" mais que M. S. Hussein commet une "erreur en espérant diviser les cinq membres du Conseil de Sécurité".

29.10.90 Le Conseil de Sécurité adopte (13 pour, pas d'opposition, Cuba et Yemen s'abstiennent) une résolution qui condamne l'occupation du Koweit par l'Irak, la prise d'otages et le mauvais traitement réservé à la population du Koweit. L'Irak sera tenu pour responsable des dégats et des violations du droit international.

30.10.90 La réunion annuelle des ministres des Affaires étrangères de l'ASEAN tenue à Jakarta énonce la menace d'un recours au boycott des produits de la CEE si celle-ci interdit l'importation de produits de la forêt tropicale de l'Asie du sud-est.

30.10.90 La République fédérative soviétique de Kirghizie est la dernière des 15 Républiques qui forment l'URSS à adopter une déclaration de souveraineté.

31.10.90 L'ambassade de l'URSS à Hanoi déclare que l'URSS a commencé à retirer ses forces de la base de Cam Ranh au Vietnam.

01.11.90 L'agence de presse Xinhua déclare que la R.P. de Chine réagira à tout acte qui viole sa souveraincté.

01.11.90 Le Vietnam annonce qu'il a réduit ses forces armées de moitié depuis 1987. La base de Cam Ranh après le retrait de la flotte soviétique sera ouverte aux navires de toutes nationalités pour des usages d'ordre économique.

02.11.90 Un projet de loi est adopté à Taiwan à propos des relations avec la R.P. de Chine qui permet des contacts civils entre les deux parties de la Chine. 
Un conseil des affaires continentales est constitué à Taipei pour servir de canal aux relations.

02.11.90 Le Japon annonce la réactivation de son programme de prêts au développement à la R.P. de Chine.

02.11.90 Visite à Vientiane du Premier ministre de Thailande. Il discute avec son homologue laotien de l'application du communiqué sur les échanges et de la paix aux frontières entre les deux pays.

03.11.90 Le front pour l'indépendance de Timor Oriental (Fretilin) offre au gouvernement d'Indonésie d'ouvrir inconditionnellement des conversations de paix. Le ministre indonésien des Affaires étrangères déclare ne pas avoir à répondre à cette offre puisque l'énorme majorité de la population de Timor Oriental a opté pour l'intégration à l'Indonésie.

3-4.10.90 Première rencontre intergouvernementale depuis 1950 entre Japonais et Nord-Coréens en vue de l'établissement de relations diplomatiques.

Déjà le 26.09.90, une délégation parlementaire japonaise avait été reçue par le Président Kim Il-sung.

06.11.90 Le Nicaragua est le 28ème état à reconnaître Taiwan en rétablissant les relations diplomatiques rompues en 1985. La R.P. de Chine suspend ses relations diplomatiques avec Managua.

Une reconnaissance de facto de deux Chines existe par les bureaux consulaires de Taiwan à l'étranger et par l'importance des relations substantielles entretenues avec Taiwan par des pays qui ont des relations diplomatiques avec Pékin.

La R.P. de Chine par ailleurs cesse de rompre ses relations diplomatiques dans les cas de reconnaissance de Taiwan; elle se borne a les suspendre.

6-13.11.90 Le Ministre des Affaires Etrangères de la R.P. de Chine en visite en Egypte, Koweit, Jordanie, Arabie Saoudite et Irak. Il considère qu'il faut une solution juste a la crise sur la base des résolutions du Conseil de Sécurité de l'ONU. 
06.11.90 A l'issue d'un débat entamé le 21 septembre, le Parlement japonais rejette le projet du Premier ministre concernant l'envoi d'un corps militaire de paix dans le Golfe.

La R.P. de Chine, la Corée du Sud, Singapour et les Philippines ont manifesté leur désapprobation à ce projet.

07.11.90 Les Etats-Unis décident de retirer en 1991 les appareils de leur armée de l'air et plus de $10 \%$ des militaires stationnés dans les bases aux Philippines.

7-13.11.90 Une délégation du Vatican négocie au Vietnam la nomination de cinq nouveaux évêques.

09.11.90 Rencontre internationale sur le Cambodge à Jakarta. 23-25 novembre les co-présidents de la conférence internationale de Paris (Indonésie et France) mettent au point un accord avec les représentants des quatre membres du Conseil de Sécurité de l'ONU. La R.P. de Chine déclare qu'elle n'a plus fourni d'armes aux forces de la résistance depuis août 1990.

24.11, le Premier ministre de Phnom Penh, M. Hun Sen, indique que son gouvernement rejette le plan de paix du Conseil de Sécurité d'août 1990. Il n'accepte pas son démantèlement ni celui de ses forces armées et considère que le rôle de l'ONU doit se borner à surveiller les élections.

09.11.90 Le roi Birendra du Népal promulgue la nouvelle Constitution; elle reconnait les droits humains fondamentaux, instaure un régime parlementaire, le multipartisme et l'hindouisme comme religion nationale.

9-10.11.90 Visite officielle de M. Gorbatchev en R.F. d'Allemagne. Signature d'un traité de bon voisinage et de coopération entre les deux pays.

11.11.90 Premières conversations entre délégués officiels de la Corée du Nord et du Japon à Pékin en vue de l'établissement de liens diplomatiques.

Deux obstacles subsistent : le Japon veut une inspection internationale des installations nucléaires de Corée du Nord. Celle-ci insiste sur des compensations financières pour les 
hostilités japonaises subies depuis 1910 alors que le Japon est disposé à ne reconnaître que la colonisation de la Corée de 1910 à 1945 .

17.11.90 Au cours des deuxièmes conversations à Pékin, le Japon lève à partir d'avril 1991, les restrictions qu'il imposait aux voyages vers la Corée du Nord.

11.11.90 Le Ministre de la culture de l'URSS en visite à Taiwan. Bien que "privées" les conversations menées constituent le premier contact depuis quarante ans de l'URSS avec Taiwan.

27-29.11 le maire de Moscou discute a Taiwan de l'établissement de liens économiques et culturels.

11.11.90 Le Secrétaire général du P.C. vietnamien affirme l'intention du Vietnam d'ouvrir la base aéro-navale de Cam Ranh aux EtatsUnis et au Japon.

12.11.90 Cérémonies à Tokyo d'intronisation de l'Empereur Akihito en présence de représentants de 158 pays.

13.11.90 Un accord est signé à Tokyo entre M. Lee Kuan Yew, Premier ministre de Singapour et $M$. Dan Quayle, Vice président des Etats-Unis sur l'utilisation accrue par les Etats-Unis des facilités militaires de Singapour.

13.11.90 De nouveaux contrôles sont imposés à la presse étrangère à Singapour.

14.11.90 Visite d'une délégation de l'ONU à Timor Oriental.

14.11.90 Traité entre la RFA et la Pologne qui confirme les frontières définies entre les deux pays le long de l'Oder et de la Neisse.

14-16.11.90 Négociations à Washington entre Ministres de la défense américain et sud-coréen. Toute réduction des forces américaines en Corée du Sud devra être graduelle et basée sur une évaluation serrée de la sécurité dans la région.

14-21.11.90 Le Premier ministre de la R.P. de Chine, M. Li Peng en visite dans trois pays d'Asie. 
Il signe trois accords de coopération économique avec le Pakistan auquel son gouvernement accorde une aide financière.

$\mathrm{Au}$ Bangladesh, M. Li Peng conclut quatre accords de coopération et un accord commercial 1990-1994. Un accord d'aide économique est signé avec le Népal auquel le gouvernement de la R.P. de Chine a fourni des armes pour sa défense nationale.

14-16.11.90 Visite en R.P. de Chine du Président de l'Argentine, M. Carlos Menem.

14-19.11.90 Visite d'état en R.P. de Chine du Président de la République d'Indonésie. Du 19 au 21.10, M. Suharto est l'hôte du Vietnam où il signe des accords de coopération économique, scientifique et technique.

16.11.90 La R.P. de Chine établit des relations diplomatiques avec les lles Marshall.

17.11.90 La R.P. De Chine dévalue de $9.57 \%$ le Remminbi. Le taux est fixé à $1 \$$ US $=5.23$ yuans.

17-18.10.90 Deuxième rencontre des Premiers ministres des deux Corée, à Pyongyang. Une prochaine rencontre aura lieu à Séoul du 11 au 14.12.90.

19-21.11.90 Les chefs d'Etat et de gouvernement des 34 participants a la Conférence sur la sécurité et la coopération en Europe, réunis à Paris, attribuent a l'Albanie un statut d'observateur et adoptent la Charte de Paris pour une nouvelle Europe ainsi que le traité sur les forces armées conventionnelles en Europe signé le 19.11 par les dirigeants des 16 membres de l'OTAN et les 6 membres du Pacte de Varsovie.

20.11.90 Le Gouvernement japonais décide de fournir une aide humanitaire de deux milliards de yens à l'URSS.

20.11.90 L'Agence Yonhap de Corée du Sud rapporte qu'à un séminaire économique tenu à Séoul, M. V. Medvdeyev a déclaré que les 
pays de la région Asie-Pacifique devraient forger une alliance économique comme contrepoids au bloc des pays industrialisés de l'Occident. Ceci est considéré comme un appui soviétique à la proposition de Séoul de faire un bloc économique entre les deux Corée, la R.P. de Chine, l'URSS et le Japon.

21.11.90 La R.P. de Chine rappelle son ambassadeur des Philippines pour exprimer son mécontentement à propos de l'attitude du gouvernement de Manille à l'égard de Taiwan.

21.11.90 Des personnalités de l'opposition afghane ont des conversations à Genève avec le Président Afghan Najibullah en vue de trouver une solution à la guerre civile qui continue dans le pays.

22.11.90 5ème sommet aux Iles Maldives, des dirigeants des sept pays membres de l'association pour la coopération régionale en Asie du Sud (Maldives, Bangladesh, Bhutan, Inde, Népal, Pakistan et Sri Lanka).

26.11.90 M. Lee Kuan Yew, Premier ministre de Singapour depuis 1959 cède son poste gouvernemental à $\mathrm{M}$. Goh Chok Tong mais conserve la direction du parti au pouvoir (P.A.P.).

27.11.90 Le Président du Bangladesh déclare l'état d'urgence après plusieurs semaines de manifestations anti-gouvernementales.

28.11.90 Le Ministre des Affaires Etrangères de la R.P. de Chine est reçu a Washington, ce qui représente la fin de l'isolement diplomatique imposé après la répression du "printemps de Pékin" du 4.6.89.

28.11.90 Les relations diplomatiques entre la Grande Bretagne et la Syrie, rompues en 1986, sont rétablies.

29.11.90 Le Conseil de Sécurité de l'ONU adopte par 12 voix contre 2 (Cuba et Yemen) et 1 abstention (R.P. de Chine) sa 12ème résolution $\left(n^{\circ} 678\right)$ à propos de l'Irak. Elle autorise le recours à 
la force contre l'Irak après le 15 janvier 1991 pour le contraindre à évacuer le Koweit.

30.11.90 La nouvelle Constitution qui entre en vigueur au Mozambique prévoit l'instauration du multipartisme et d'une économie de marché.

04.12.90 La pression populaire contraint le Général Ershad a la démission. Au pouvoir au Bangladesh depuis 1982, il est arrêté le 12.12 .

06.12.90 M. Saddam Hussein annonce la libération des otages occidentaux et japonais encore retenus en Irak.

Les otages soviétiques sont également autorisés à quitter l'Irak, après les deux visites de l'envoyé spécial de $M$. Gorbatchev à Bagdad les 4 et 28 octobre.

06.12.90 Un accord sur la pèche est signé entre l'URSS et la Nouvelle Guinée-Papouasie.

07.12.90 La CEE lance un appel à la clémence aux autorités de la R.P. de Chine où se déroulent des procès politiques, en particulier d'étudiants accusés d'activités contre-révolutionnaires.

07.12.90 Les travaux de la Conférence ministérielle de l'Uruguay Round sont suspendus à Bruxelles. Le désaccord persiste entre la CEE et les Etats-Unis : la CEE propose une réduction de $30 \%$ de son programme d'aide à l'agriculture de 1986 a 1996 alors que les Etats-Unis proposent $75 \%$ de réduction (sur chiffres de 1986-88) de l'appui interne aux agriculteurs européens et $90 \%$ de réduction des subsides à l'exportation de la CEE.

09.12.90 M. Lech Walesa devient le premier Président de la République de Pologne élu au suffrage universel.

10-14.12.90 Le Premier Ministre de R.P. de Chine en visite en Malaysie. Le Premier ministre malais propose le renforcement des liens économiques entre pays de la région pour s'opposer aux blocs économiques. 
Aux Philippines, M. Li Peng déclare que la R.P. de Chine ne s'oppose pas aux liens non officiels noués avec Taiwan.

11.12.90 Le pluralisme est accepté en Albanie. Le 21.12, la déstalinisation est décrétée; le 26.12, le P.C. renonce au centralisme démocratique.

12.12.90 M. Bush annonce un crédit de 1 milliard de $\$$ des Etats-Unis a l'URSS pour l'achat de produits agricoles américains.

14.12.90 Le Président de la Corée du Sud entame une visite officielle en URSS.

14-15.12.90 Le Conseil européen réuni à Rome décide une aide alimentaire d'urgence à l'URSS.

18.12.90 Le Japon octroye $100 \mathrm{~m}$. \$ de crédits à l'URSS à titre d'assistance humanitaire.

Le 28.11.90, il avait offert une aide médicale d'urgence à l'URSS.

20.12.90 M. E. Chevardnadze, Ministre des Affaires étrangères de l'URSS depuis 1985 démissionne et met en garde contre le retour de la dictature.

21-23.12.90 Conférence à Paris sur le Cambodge. Le Conseil national suprême accepte les points principaux du document sur le réglement de paix, les partics sont invitées à la retenue dans les combats.

24.12.90 M. Gorbatchev obtient du Congrès des députés de l'URSS l'approbation de ses projets de referendum sur l'appartenance des Républiques à l'Union et sur le statut de la terre.

29.12.90 La Corée du Nord nie avoir envoyé des conseillers militaires en Irak. 
31.12.90 Clôture du 7ème plenum du 13eme C.C. du P.C. chinois. La session a adopté les propositions du plan décennal et le 8̀me plan quinquennal. Il réaffirme la politique de réforme et d'ouverture destinéc à développer le système socialiste.

La restructuration doit encore durer deux ans; la réforme des prix est mise à l'ordre du jour. 